\title{
Tight Bounds for Restricted Grid Scheduling*
}

\author{
Joan Boyar \\ Department of Mathematics and Computer Science, University of Southern Denmark, \\ Campusvej 55, DK-5230 Odense M, Denmark \\ joan@imada.sdu.dk \\ Faith Ellen \\ Department of Computer Science, University of Toronto, \\ 10 King's College Road, Toronto, Ontario, Canada M5S $3 G 4$ \\ faith@cs.toronto.edu \\ Received (Day Month Year) \\ Accepted (Day Month Year) \\ Communicated by (xxxxxxxxxx)
}

\begin{abstract}
The following problem is considered: Items with integer sizes are given and variable sized bins arrive online. A bin must be used if there is still an item remaining which fits in it when the bin arrives. The goal is to minimize the total size of all the bins used. Previously, a lower bound of $\frac{5}{4}$ on the competitive ratio of this problem was achieved using items of size $S$ and $2 S-1$. For these item sizes and maximum bin size $M=4 S-3$, we obtain asymptotically matching upper and lower bounds, which vary depending on the ratio of the number of small items to the number of large items.

Keywords: Online algorithms; variable-sized bin packing; Restricted Grid Scheduling.
\end{abstract}

\section{Introduction}

In the classical online bin packing problem, bins of unit size are given and items of varying size, each at most $M$, arrive online. This is reversed in the Grid Scheduling problem 2], where items and their sizes are given and bins of varying size arrive online. In this problem, a bin must be used if there is at least one unpacked item that fits in it. As in the classical problem, the items must all be packed in the bins,

*A preliminary version of this paper, entitled "Bounds for Scheduling Jobs on Grid Processors", appeared in Space-Efficient Data Structures, Streams, and Algorithms, Lecture Notes in Computer Science, volume 8066, 2013, pages 12-26. 


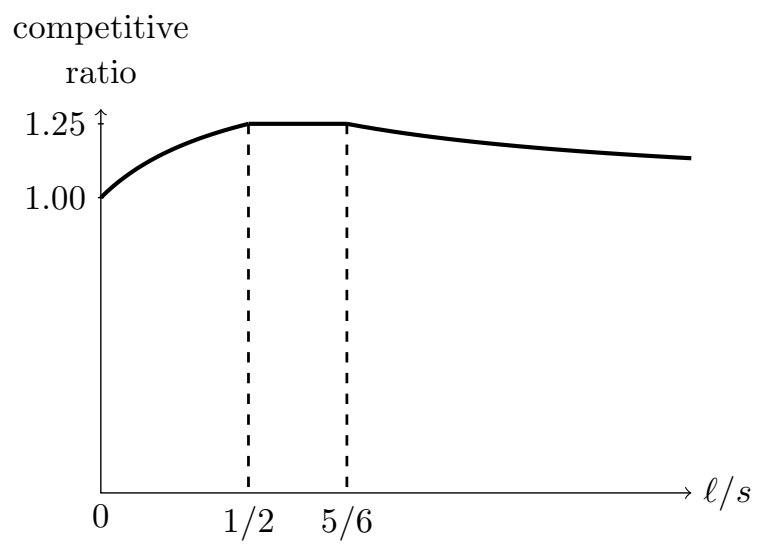

Fig. 1. The competitive ratio of the Restricted Grid Scheduling problem as a function of $\ell / s$

so that the total size of the items packed in a bin is no more than the size of the bin. The goal is to minimize the sum of the sizes of bins used to pack the items: Bins which were unused because no unpacked items fit in them do not contribute to this total. It is assumed that there are plenty of bins that are large enough to hold the largest items, so that feasibility is not an issue.

The Grid Scheduling problem is known to have competitive ratio at least $\frac{5}{4}$ [2]. This was achieved using items of size $S$ and $L=2 S-1$, for integers $S \geq 2$. In this paper, we consider the Restricted Grid Scheduling problem, where only these item sizes are allowed and the maximum possible bin size is $M=4 S-3$. For this restricted problem, we obtain asymptotically matching upper and lower bounds, which depend on the ratio of $s$, the number of small items, to $\ell$, the number of large items. Specifically, if $s$ items of size $S>1$ and $\ell$ items of size $L=2 S-1$ are given and the maximum bin size is $M=4 S-3$, the competitive ratio for this problem is

$$
\begin{array}{ll}
1+\frac{1}{2+s / \ell} & \text { if } \ell \leq s / 2, \\
1+\frac{1}{4} & \text { if } s / 2<\ell \leq 5 s / 6, \text { and } \\
1+\frac{2}{3+6 \ell / s} & \text { if } 5 s / 6<\ell .
\end{array}
$$

This bound is plotted in Figure 1

The Grid Scheduling Problem The Grid Scheduling problem [2, 3, 5, was proposed in an article concerning a Grid architecture [11. The proposed application in that article concerned the well-known bioinformatics program BLAST. More generally, one considers a large problem that is divided up into independent jobs, which may be of varying sizes. These jobs are distributed to heterogeneous processors (for example, personal computers) having idle time. These processors become available in an on-line manner, as they become idle. Folding@Home (in which idle processors 
are used to find new ways to fold proteins) and SETI@home (in which idle processors are used to look for signs of extraterrestrial life) are two other examples. Such systems have been called Grids, denoting computation distributed seamlessly over (possibly) large distances, in the same way that electricity is distributed over the electrical grid. It does not refer to computation in which the network topology is a rectangular grid. Note that other researchers have used the terminology "Grid scheduling" to denote other problems which are very different from the one considered here.

It is well-known that paging may slow down computation drastically. In fact, parallelizing jobs can result in superlinear speed-up by eliminating unnecessary paging [4. Paging can be avoided by assigning jobs to a processor whose memory capacity is at least the combined memory requirements of those jobs. In the Grid Scheduling problem, the item sizes are the memory requirements of the jobs and the bin sizes are the memory capacity of the processors. To finish the jobs as quickly as possible, any processor that arrives and can process at least one of the remaining jobs should be assigned to it (rather than waiting for a processor of close to the right size, which might never come). For the Grid Scheduling problem, this condition is equivalent to requiring that any arriving bin should be used, if there is an unpacked item that fits into it. We prove, in fact, that items should be packed into an arriving bin until there are no more items that can fit into it. Allowing bins which are smaller than the maximum item size reflects the situation where some processors might not have enough memory to handle the largest jobs.

Related Work In [2, Boyar and Favrholdt presented an algorithm to solve the Grid Scheduling problem with competitive ratio $\frac{13}{7}$. They also proved that the competitive ratio of any algorithm for the Grid Scheduling problem is at least $\frac{5}{4}$. In that proof, the adversary used only two different item sizes, $S$ and $L=2 S-1$, and four different bin sizes, $S, 2 S-1,2 S$, and $4 S-4$, for an integer $S>1$.

This $\frac{5}{4}$ lower bound bound has not yet been improved, despite its distance from the $\frac{13}{7}$ upper bound. Here, we contribute to understanding why improving the lower bound may be difficult: We show that the lower bound is tight in the sense that there is an algorithm with a competitive ratio of $\frac{5}{4}$ when there are only items of size $S$ and $L=2 S-1$ and the maximum bin size is $4 S-3$. In addition to allowing all possible bin sizes in this range, we consider all possible ratios of the number of items of size $S$ to the number of items of size $L$ and show that the competitive ratio is $\frac{5}{4}$ in a middle range, but decreases as the ratio moves away from this middle range. The bounds obtained are tight. Some of the results concerning packings are general, not restricted to this special case, and might be useful in narrowing the gap between the upper and lower bounds for the general problem.

Grid Scheduling with Conflicts, the Grid Scheduling problem with the restriction that certain pairs of jobs cannot be executed on the same processor (i.e. be placed in the same bin), was later studied by Epstein et al. in [5]. In the semi-online version, 
where the bins come in non-increasing order, they show a lower bound of $\frac{5}{4}$ and an upper bound of 3 on the competitive ratio.

In Zhang's Bin Packing problem [12, it is also the case that items are given in advance and bins arrive online. Bins and items can have any sizes in the range $(0,1]$, but the smallest bin is at least as large as the largest item, so that each item can be packed in any bin. Zhang showed that analogues of four classical bin packing algorithms, including First-Fit Decreasing, all have a competitive ratio of 2. Algorithms for the Grid Scheduling problem also apply to Zhang's Bin Packing problem with the same competitive ratio. In particular, the algorithm in [2, with a competitive ratio of $\frac{13}{7}$, solves an open question proposed in [12, asking if there exists an algorithm with competitive ratio less than 2. However, lower bounds for the Grid Scheduling Problem do not apply to Zhang's Bin Packing problem, since the Grid Scheduling problem does not restrict the sizes of the bins to be at least as large as every item.

In many applications of bin packing, there are only a small number of different item sizes. A number of papers have considered the problem of packing a sequence of items of two different sizes in bins of size 1 in an online manner. In particular, there is a lower bound of $4 / 3$ on the competitive ratio [7,9] and a matching upper bound $[7$. When both item sizes are bounded above by $1 / k$, the competitive ratio can be improved to $\frac{(k+1)^{2}}{k^{2}+k+1}[$ ]

Overview We begin with some preliminaries, including a formal definition of the Grid Scheduling problem. In Section 3 , we prove the lower bound on the competitive ratio of the Restricted Grid Scheduling problem (and, hence, of the Grid Scheduling problem). Then we provide a matching upper bound for the Restricted Grid Scheduling problem, under the assumption that the maximum possible bin size, $M$, is at most $4 S-3$. As discussed in Section 2, one has to assume that $M$ is bounded (as compared to $S$ ) in the general Grid Scheduling problem for any algorithm to be competitive. Thus, our algorithm shows that any better lower bound for the general Grid Scheduling problem must use more than two item sizes, different item sizes, or a larger maximum bin size. In Section 4 we present properties of optimal packings for the Restricted Grid Scheduling problem. Some of these provide motivation for the design of our algorithm, while others are important for the analysis. We show why a few simple algorithms are not optimal in Section 5, Our algorithm, 2-Phase-Packer, appears in Section 6, together with the analysis. In Section 7, we consider a slightly relaxed version of the Grid Scheduling problem and show that it is equivalent to the version we defined. We conclude with some open questions.

\section{Preliminaries}

Given a set of items, each with a positive integer size, and a sequence of bins, each with a positive integer size at most $M$, the goal of the Grid Scheduling problem is 
to pack all the items in the bins so that the sum of the sizes of the items packed in each bin is at most the size of the bin and the sum of the sizes of bins used is minimized. The bins in the sequence arrive one at a time and each must be packed before the next bin arrives, without knowledge of the sizes of any future bins. If a bin is at least as large as the smallest unpacked item, it must be packed with at least one item. Moreover, after it has been packed, no unpacked item fits into the space that remains in the bin. There is no cost for a bin that is smaller than the smallest unpacked item. Note, there is no loss of generality in assuming that every item has size at most $M$, because no item of size greater than $M$ can be packed.

Because all items are required to be packed, it is assumed that enough sufficiently large bins arrive. Thus, any algorithm eventually packs all items. For example, it suffices that every sequence has a suffix consisting of bins of size $M$, whose length is equal to the number of items.

The cost of a packing is the sum of the sizes of the bins it uses (i.e. in which it places at least one item). A packing is optimal if it is feasible and its cost is not more than that of any other feasible packing for the same set of items and sequence of bins.

The competitive ratio [1,8,10, of an on-line algorithm is the worst-case ratio of the on-line performance to the optimal off-line performance, up to an additive constant. More precisely, for a set $I$ of items (or, equivalently, a multi-set of item sizes), a sequence $\sigma$ of bins, and an algorithm $\mathbb{A}$ for the Grid Scheduling problem, let $\mathbb{A}(I, \sigma)$ denote the cost of the packing produced by $\mathbb{A}$ when packing $I$ in the sequence $\sigma$ of bins. Then, the competitive ratio $\mathrm{CR}_{\mathbb{A}}$ of $\mathbb{A}$ is

$$
\mathrm{CR}_{\mathbb{A}}=\inf \{c \mid \exists d, \forall I, \forall \sigma, \mathbb{A}(I, \sigma) \leq c \cdot \mathrm{OPT}(I, \sigma)+d\},
$$

where $\operatorname{OPT}(I, \sigma)$ denotes the minimum cost of any feasible packing of $I$ in the sequence $\sigma$ of bins i.e. produced by an optimal off-line algorithm. For specific choices of families of increasingly large sets $I_{n}$ and sequences $\sigma_{n}$, with $n \in \mathbb{N}$, the performance ratios, $\frac{\mathbb{A}\left(I_{n}, \sigma_{n}\right)}{\operatorname{OPT}\left(I_{n}, \sigma_{n}\right)}$, can be used to prove a lower bound on the competitive ratio of $\mathbb{A}$.

If there is no bound on the maximum bin size, the Grid Scheduling problem is uninteresting, because the competitive ratio is unbounded: Once enough bins for an optimal packing have arrived, an adversary could give bins of arbitrarily large size, which the algorithm would be forced to use. This can be seen in the following example: Suppose there are two items of size $S$ and one of size $L=2 S-1$. The first bin has size $2 S$. If the algorithm puts at least one of the small items there, the adversary next gives two bins of size $S$, followed by one of size $M$. If the algorithm puts the larger item in the first bin, the adversary next gives one bin of size $L$, followed by one of size $M$. The ratio of the algorithm's performance compared to the adversary's is at least $\min \{(2 S+M) / 4 S,(2 S+L+M) /(2 S+L)\}$. This is close to $\frac{3}{2}$ when $M=4 S-3$, but it is unbounded if $M$ can be arbitrarily large compared 
to $S$. Note that this example also shows a lower bound of $\frac{3}{2}$ on the strict competitive ratio (the competitive ratio where the additive constant in the definition is zero), even when $M=4 S-3$, but not on the competitive ratio.

The Restricted Grid Scheduling problem is the restriction of the Grid Scheduling problem in which all items have size $S$ or $L=2 S-1$, where $M=4 S-3$ and $S>1$. Note that the maximum size $M=4 S-3$ was chosen to be as large as possible without allowing space for two items of size $L$. There is no loss of generality in assuming that every bin has size at least $S$, because no bin of size less than $S$ can be used to pack an item. The value $S$ is assumed to be a constant, to allow an algorithm to use one extra bin of size $M$, for example, which is only counted in the additive constant of the competitive ratio. The definition of competitive ratio allows the additive constant to be arbitrarily large, as long as it is independent of the number of items.

\section{Lower Bounds}

In this section, we assume that there can be an unbounded number of items of size $S$ and an unbounded number of items of size $L$. Otherwise, an algorithm that gives lower preference to the items of which there are only a bounded number, has competitive ratio 1 , taking the additive constant to be $d=\min \{s, \ell\} \cdot M$.

Theorem 1. No algorithm for the Restricted Grid Scheduling problem has competitive ratio lower than

$$
\begin{cases}1+\frac{1}{2+s / \ell} & \text { if } \ell \leq s / 2, \\ 1+\frac{1}{4} & \text { if } s / 2<\ell \leq 5 s / 6, \text { and } \\ 1+\frac{2}{3+6 \ell / s} & \text { if } 5 s / 6<\ell\end{cases}
$$

Proof. Consider an algorithm for the Restricted Grid Scheduling problem and an instance in which there are $s$ items of size $S>1$, $\ell$ items of size $L=2 S-1$, and maximum bin size $M=4 S-3$. We start with the case when $\ell \leq s / 2$ and then handle the case when $\ell>s / 2$. In both cases, we consider two subcases, depending on how the algorithm packs the first batch of bins.

Case I: $\ell \leq s / 2$.

The adversary begins by giving $\ell$ bins of size $2 S$. In each of these bins, the algorithm must pack either two items of size $S$ or one item of size $L$. Let $0 \leq k \leq \ell$ be the number of these bins in which the algorithm packs two items of size $S$. Then the algorithm has $s-2 k$ items of size $S$ and $k$ items of size $L$ left to pack.

Case I.1: $k \leq \ell / 2$.

Next, the adversary gives $s-2 \ell$ bins of size $S$, followed by $2 \ell$ bins of size $L$. The 
algorithm must pack one item of size $S$ in each bin of size $S$ and must use one bin of size $L$ for each of the remaining $s-2 k-(s-2 \ell)=2(\ell-k)$ items of size $S$ and $k$ items of size $L$. The total cost incurred by the algorithm is $\ell \cdot 2 S+(s-2 \ell) \cdot S+(2 \ell-k) \cdot L=$ $s \cdot S+2 \ell \cdot L-k \cdot L \geq s \cdot S+3 \ell \cdot L / 2$.

For this sequence, OPT packs two items of size $S$ in each of the $\ell$ bins of size $2 S$, one item of size $S$ in each of the $s-2 \ell$ bins of size $S$, and one item of size $L$ in each of the next $\ell$ bins of size $L$, for total cost $s \cdot S+\ell \cdot L$. Thus, the performance ratio of the algorithm is at least

$\frac{s \cdot S+3 \ell \cdot L / 2}{s \cdot S+\ell \cdot L}=1+\frac{\ell \cdot L}{2 \ell \cdot L+2 s \cdot S}=1+\frac{1}{2+\frac{s}{\ell}+\frac{s / \ell}{2 S-1}} \rightarrow 1+\frac{1}{2+s / \ell}$ as $S \rightarrow \infty$.

Case I.2: $k>\ell / 2$.

Next, the adversary gives $s$ bins of size $S$, followed by $\ell$ bins of size $M$. The algorithm packs one item of size $S$ in the first $s-2 k \geq 2 \ell-2 k \geq 0$ of these bins, using up all its items of size $S$. It discards the remaining $2 k$ bins of size $S$, because it has no remaining elements that are small enough to fit in them. Then the algorithm packs its remaining $k$ items of size $L$ into $k$ bins of size $M=4 S-3$. The total cost incurred by the algorithm is

$\ell \cdot 2 S+(s-2 k) \cdot S+k \cdot(4 S-3)=(2 \ell+s) \cdot S+k \cdot(2 S-3)>(2 \ell+s) \cdot S+\ell \cdot(S-3 / 2)$.

For this sequence, OPT packs one item of size $L$ in each of the $\ell$ bins of size $2 S$ and one item of size $S$ in each of the next $s$ bins. The total cost of OPT's packing is $\ell \cdot 2 S+s \cdot S$. Thus, the performance ratio of the algorithm is greater than

$$
\frac{(3 \ell+s) \cdot S-3 \ell / 2}{(2 \ell+s) \cdot S}=1+\frac{1-3 /(2 S)}{2+s / \ell} \rightarrow 1+\frac{1}{2+s / \ell} \text { as } S \rightarrow \infty .
$$

Case II: $\ell>s / 2$.

The adversary begins by giving $\lfloor s / 2\rfloor$ bins of size $2 S$. In each of these bins, the algorithm must pack either two items of size $S$ or one item of size $L$. Let $0 \leq k \leq$ $\lfloor s / 2\rfloor$ be the number of these bins in which the algorithm packs two items of size $S$. Then the algorithm has $s-2 k$ items of size $S$ and $\ell-\lfloor s / 2\rfloor+k$ items of size $L$ left to pack.

Case II.1: $k \leq\lfloor s / 2\rfloor-s / 8-\ell / 4+1$ or $k \leq\lfloor s / 2\rfloor-s / 3+1$.

Next, the adversary gives $\lceil s / 2\rceil-\lfloor s / 2\rfloor$ bins of size $S$ (i.e. one bin of size $S$ if $s$ is odd and no bins of size $S$ if $s$ is even), $\lfloor s / 2\rfloor-k+\ell-1$ bins of size $L$, and one bin of size $M$. Since $(s-2 k)+(\ell-\lfloor s / 2\rfloor+k)=(\lceil s / 2\rceil-\lfloor s / 2\rfloor)+(\lfloor s / 2\rfloor-k+\ell-1)+1$, the algorithm packs one of its remaining items in each of these bins, so the total cost it incurs is $\lfloor s / 2\rfloor \cdot 2 S+(\lceil s / 2\rceil-\lfloor s / 2\rfloor) \cdot S+(\lfloor s / 2\rfloor-k+\ell-1) \cdot L+M=$ $s \cdot S+\ell \cdot L+(\lfloor s / 2\rfloor-k+1) \cdot L-1$.

We may assume, without loss of generality, that $s \geq 4$. Then $\lfloor s / 2\rfloor-k \geq \min \{s / 8+$ $\ell / 4, s / 3\}-1>s / 4-1 \geq 0$, so there are at least $\ell$ bins of size $L$. For this sequence, 
OPT packs two items of size $S$ in each of the $\lfloor s / 2\rfloor$ bins of size $2 S$, one item of size $S$ in the bin of size $S$, if $s$ is odd, and one item of size $L$ in each of the next $\ell$ bins of size $L$. Its total cost is $s \cdot S+\ell \cdot L$.

If $k \leq\lfloor s / 2\rfloor-s / 8-\ell / 4+1$, then $(\lfloor s / 2\rfloor-k+1) \cdot L \geq(s / 8+\ell / 4) \cdot L=S \cdot s / 4-$ $s / 8+L \cdot \ell / 4$, so the performance ratio of the algorithm is at least

$$
\frac{(s \cdot S+\ell \cdot L) \cdot 5 / 4-s / 8-1}{s \cdot S+\ell \cdot L}=\frac{5}{4}-\frac{s / 8+1}{s \cdot S+\ell \cdot(2 S-1)} \rightarrow \frac{5}{4} \text { as } S \rightarrow \infty .
$$

Similarly, if $k \leq\lfloor s / 2\rfloor-s / 3+1$, then $(\lfloor s / 2\rfloor-k+1) \cdot L \geq(s / 3) \cdot L$, so the performance ratio of the algorithm is at least

$$
1+\frac{(s / 3) \cdot L}{s \cdot S+\ell \cdot L}=1+\frac{2-1 / S}{3+6 \ell / s-3 \ell /(s \cdot S)} \rightarrow 1+\frac{2}{3+6 \ell / s} \text { as } S \rightarrow \infty
$$

Case II.2: $k>\lfloor s / 2\rfloor-s / 8-\ell / 4+1$ and $k>\lfloor s / 2\rfloor-s / 3+1>s / 6$.

Next, the adversary gives $\max \{s-2 k, s-\ell+\lfloor s / 2\rfloor\}$ bins of size $S$, followed by $\min \{2 k, \ell-\lfloor s / 2\rfloor\}$ bins of size $L+S, \max \{\ell-\lfloor s / 2\rfloor-2 k, 0\}$ bins of size $L$, and finally $k$ bins of size $M=4 S-3$. The algorithm packs its $s-2 k$ items of size $S$ into bins of size $S$. Since $\min \{2 k, \ell-\lfloor s / 2\rfloor\}+\max \{\ell-\lfloor s / 2\rfloor-2 k, 0\}+k=\ell-\lfloor s / 2\rfloor+k$, the algorithm packs one item of size $L$ in each bin of size $L+S, L$, and $M$. The total cost incurred by the algorithm is

$$
\begin{aligned}
& \lfloor s / 2\rfloor \cdot 2 S+(s-2 k) \cdot S+\min \{2 k, \ell-\lfloor s / 2\rfloor\} \cdot(L+S) \\
& \quad+\max \{\ell-\lfloor s / 2\rfloor-2 k, 0\} \cdot L+k \cdot M \\
& =(2\lfloor s / 2\rfloor+s) \cdot S+(\ell-\lfloor s / 2\rfloor) \cdot L+k \cdot(2 S-3)+\min \{2 k, \ell-\lfloor s / 2\rfloor\} \cdot S .
\end{aligned}
$$

For this sequence, OPT fills every bin it uses except for the $\lfloor s / 2\rfloor$ bins of size $2 S$, in which it puts items of size $L=2 S-1$. Therefore, the total cost of OPT's packing is $s \cdot S+\ell \cdot L+\lfloor s / 2\rfloor$.

If $2 k \geq \ell-\lfloor s / 2\rfloor$, the performance ratio of the algorithm is at least

$$
\begin{aligned}
& \frac{(2\lfloor s / 2\rfloor+s) \cdot S+(\ell-\lfloor s / 2\rfloor) \cdot L+k \cdot(2 S-3)+(\ell-\lfloor s / 2\rfloor) \cdot S}{s \cdot S+\ell \cdot L+\lfloor s / 2\rfloor} \\
& >\frac{(\ell+s+\lfloor s / 2\rfloor) \cdot S+(\ell-\lfloor s / 2\rfloor) \cdot L+(\lfloor s / 2\rfloor-s / 8-\ell / 4+1) \cdot(2 S-3)}{s \cdot S+\ell \cdot L+\lfloor s / 2\rfloor} \\
& =\frac{5}{4}+\frac{(\lfloor s / 2\rfloor-s / 2+2) \cdot S+\ell+3 s / 8-13\lfloor s / 2\rfloor / 4-3}{(s+2 \ell) \cdot S-\ell+\lfloor s / 2\rfloor} \\
& >\frac{5}{4}+\frac{\ell+3 s / 8-13\lfloor s / 2\rfloor / 4-3}{(s+2 \ell) \cdot S-\ell+\lfloor s / 2\rfloor} \rightarrow \frac{5}{4} \text { as } S \rightarrow \infty .
\end{aligned}
$$


If $2 k \leq \ell-\lfloor s / 2\rfloor$, the performance ratio of the algorithm is at least

$$
\begin{aligned}
& \frac{(2\lfloor s / 2\rfloor+s) \cdot S+(\ell-\lfloor s / 2\rfloor) \cdot L+k \cdot(4 S-3)}{s \cdot S+\ell \cdot L+\lfloor s / 2\rfloor} \\
& =1+\frac{k \cdot(4 S-3)}{(s+2 \ell) \cdot S-\ell+\lfloor s / 2\rfloor} \\
& >1+\frac{(s / 6) \cdot(4 S-3)}{(s+2 \ell) \cdot S+\lfloor s / 2\rfloor-\ell} \\
& =1+\frac{2-3 / 2 S}{3+6 \ell / s+3(\lfloor s / 2\rfloor-\ell) / s S} \rightarrow 1+\frac{2}{3+6 \ell / s} \text { as } S \rightarrow \infty .
\end{aligned}
$$

Note that $\frac{5}{4} \leq 1+\frac{2}{3+6 \ell / s}$ if and only if $\ell \leq 5 s / 6$. Thus, $\frac{5}{4}$ is a lower bound on the competitive ratio when $s / 2<\ell \leq 5 s / 6$ and $1+\frac{2}{3+6 \ell / s}$ is a lower bound on the competitive ratio when $\ell>5 s / 6$.

The above lower bound uses limits taken as the size, $S$, approaches infinity, despite the value $S$ being considered a constant. The point is that, for any constant $\epsilon$, there exists a value for $S$ making the lower bound greater than the actual ratio minus $\epsilon$. A similar proof gives the same ratio if one normalizes the sizes (to $M=1, S=1 / 4+\epsilon$ and $L=1 / 2+\delta$, where $0<\delta<2 \epsilon$ ) and lets $\epsilon$ approach zero. It is simply necessary that $L$ be asymptotically close to, but less than $2 S$, and $M$ be asymptotically close to, but less than $2 L$.

\section{Properties of Optimal Packings for Restricted Grid Scheduling}

From now on, we assume that there are no bins of size less than $S$. Intuitively, it seems bad to place an item of size $S$ in a bin if the algorithm could have placed an item of size $L$ there instead. We say that a bin used in a packing is bad if it contains at least one item of size $S$, it has empty space at least $L-S$, and some later bin contains an item of size $L$. Note that a bin containing an item of size $L$ and an item of size $S$ has empty space at most $M-L-S=L-S-1$, so it is not bad.

Lemma 2. For any finite set of items and any sequence of bins, there exists an optimal packing that contains no bad bin.

Proof. Let $p$ be any optimal packing of a finite set of items $I$ into a sequence of bins $\sigma=\left\langle b_{1}, b_{2}, \ldots, b_{m}\right\rangle$. Assume the claim is true for any smaller set of items.

Suppose $p$ contains a bad bin. Let $b_{f}$ be the first bad bin in $p$ and let $b_{\ell}$ be the last bin in $p$ that contains an item of size $L$. Then, by definition of bad, $f<\ell$ and, by definition of the Grid Scheduling problem, the empty space in $b_{f}$ is less than $L$.

First suppose that $p$ has an empty bin between $b_{f}$ and $b_{\ell}$. Let $b_{k}$ be the first such bin. Since $p$ is feasible, size $\left(b_{k}\right)<L$ and only items of size $L$ are packed in bins 
$b_{k}, \ldots, b_{m}$. At most one item of size $L$ can be packed in any bin (of size at most $M)$, so each nonempty bin after $b_{k}$, including $b_{\ell}$, contains exactly one item, which is of size $L$. Consider the packing $p^{\prime}$ obtained from $p$ by moving one item of size $S$ from bin $b_{f}$ to bin $b_{k}$ and moving the item of size $L$ in bin $b_{\ell}$ to bin $b_{f}$. Then $b_{\ell}$ is empty in the packing $p^{\prime}$. Since $p$ is feasible and each bin in $p^{\prime}$ (except for the unused bin, $b_{\ell}$, ) is as full as the corresponding bin in $p$, it follows that $p^{\prime}$ is also feasible. But the cost of $p^{\prime}$ is equal to $\operatorname{cost}(p)-\operatorname{size}\left(b_{\ell}\right)+\operatorname{size}\left(b_{k}\right)<\operatorname{cost}(p)$, since size $\left(b_{k}\right)<L \leq \operatorname{size}\left(b_{\ell}\right)$. This contradicts the optimality of $p$.

Therefore $p$ has no empty bins between $b_{f}$ and $b_{\ell}$. Then a packing $p^{\prime}$ can be obtained from $p$ by switching an item of size $S$ in $b_{f}$ with the item of size $L$ in $b_{\ell}$. Note that $p^{\prime}$ is optimal, since $p$ is. Since $b_{f}$ contains an item of size $L$, it is not bad in $p^{\prime}$. Since $p$ is feasible, no item packed in bin $b_{f}$ or later fits in any bin prior to $b_{f}$. The same is true for $p^{\prime}$, since $p$ and $p^{\prime}$ are the same prior to bin $b_{f}$. Furthermore, in $p^{\prime}$, no item packed in a bin after $b_{f}$ will fit in bin $b_{f}$, since its empty space, which was less than $L$ in $p$, is less than $L+S-L=S$ in $p^{\prime}$.

Let $J \subsetneq I$ be the set of items that $p^{\prime}$ packs into $\sigma^{\prime}=\left\langle b_{f+1}, \ldots, b_{m}\right\rangle$. By the induction hypothesis, there is an optimal packing $q$ of $J$ into $\sigma^{\prime}$ that contains no bad bins. Let $p^{\prime \prime}$ be the packing of $I$ into $\sigma$ that packs each item in $I-J$ into the same bin that $p^{\prime}$ does and packs each item in $J$ into the same bin that $q$ does. Then $p^{\prime \prime}$ is an optimal packing without bad bins. By induction, the claim is true for all finite sets of items, $I$.

Lemma 2 motivates the following definition.

Definition 3. A packing is reasonable if, except for those bins that arrive when there are no items of size $L$ remaining or at most two items of size $S$ remaining, every bin $b$ it uses contains

- one item of size $S$, if size $(b) \in[S, L-1]$,

- one item of size $L$, if size $(b)=L$,

- two items of size $S$ or one item of size $L$, if size $(b) \in[L+1, L+S-1]$,

- one item of size $S$ and one item of size $L$, if size $(b)=L+S$, and

- three items of size $S$ or one item of size $S$ and one item of size $L$, if $\operatorname{size}(b) \in[L+S+1,2 L-1]$.

Note that a feasible packing with no bad bins is reasonable.

Corollary 4. For any set of items and any sequence of bins, there exists an optimal packing that is reasonable.

From now on, we will restrict attention to reasonable packings. 
Given a set of items and a sequence of bins, two reasonable packings of these bins (where different subsets of the items might be packed) can differ as to whether they use one item of size $L$ or two items of size $S$ in certain bins. Therefore, the numbers of items of size $S$ and items of size $L$ they do not assign may differ. However, if both have at least one item of size $S$ and at least one item of size $L$ available, the set of bins they have used is the same and there is a simple invariant relating the numbers of available items of size $S$ and available items of size $L$ they have.

Lemma 5. Given a set of items, consider two reasonable packings of the same sequence of bins, which might pack different subsets of the items. Suppose that before bin $b$, each has at least one item of size $S$ and at least one item of size $L$ available. Then immediately after bin $b$ has been packed, the sum of the number of items of size $S$ available plus twice the number of items of size $L$ available is the same for both.

Proof. Consider any bin $b$ in the sequence $\sigma$ and suppose that immediately before bin $b$ is packed, each packing has items of both size $S$ and $L$ available and the sum of the number of items of size $S$ available plus twice the number of items of size $L$ available is the same for both. Note that this is true initially, since all items are still available for both. Since both are reasonable, either bin $b$ is filled the same way in both or in one of these packings, bin $b$ contains two more items of size $S$ and one less item of size $L$ than the other. Thus the claim remains true immediately after bin $b$ is packed.

For any sequence of bins $\sigma$ and any nonnegative integers $s$ and $\ell$, let $\operatorname{OPT}(\sigma, s, \ell)$ denote the cost of an optimal packing of $s$ items of size $S$ and $\ell$ items of size $L=2 S-1$ using $\sigma$. This must be at least the sum of the sizes of all the items.

Proposition 6. For all sequences of bins $\sigma$ and all integers $s, \ell \geq 0, O P T(\sigma, s, \ell) \geq$ $s S+\ell L$.

Given any optimal packing for a set of items, a packing for a subset of these items can be obtained by removing the additional items from bins, starting from the end.

Proposition 7. For all sequences of bins $\sigma$ and all integers $0 \leq s^{\prime} \leq s$ and $0 \leq$ $\ell^{\prime} \leq \ell, O P T\left(\sigma, s^{\prime}, \ell^{\prime}\right) \leq O P T(\sigma, s, \ell)$.

For any sequence of bins $\sigma$ and any nonnegative integers $s$ and $\ell$, let $R(\sigma, s, \ell)$ denote the maximum cost of any reasonable packing of $s$ items of size $S$ and $\ell$ items of size $L=2 S-1$ into $\sigma$.

When all items have the same size, all algorithms, including OPT, behave exactly the same. 
Proposition 8. For all sequences of bins $\sigma$ and all integers $s^{\prime}, \ell^{\prime} \geq 0, R\left(\sigma, s^{\prime}, 0\right)=$ $O P T\left(\sigma, s^{\prime}, 0\right)$ and $R\left(\sigma, 0, \ell^{\prime}\right)=O P T\left(\sigma, 0, \ell^{\prime}\right)$.

Suppose that $R$ and OPT both run out of items of size $L$ at the same time or they both run out of items of size $S$ at the same time. If they have items of the other size remaining, then, by Lemma [5, they have the same number remaining. By feasibility, they have used the same set of bins and, by Proposition 8, they will use the same set of bins for the remaining items. Thus, they have the same cost.

The following four lemmas describe the relationship between the costs incurred by $R$ and OPT when one of them has run out of one size of items. We begin with the case where OPT is the first to run out of something and it runs of items of size $L$.

Lemma 9. For all sequences of bins $\sigma$ and all integers $s^{\prime}, \ell^{\prime} \geq 0$, $R\left(\sigma, s^{\prime}, \ell^{\prime}\right) \leq O P T\left(\sigma, s^{\prime}+2 \ell^{\prime}, 0\right)+\ell^{\prime}(2 S-3)$.

Proof. by induction on $s^{\prime}$ and $\ell^{\prime}$.

If $\ell^{\prime}=0$, then, by Proposition 8, $R\left(\sigma, s^{\prime}, 0\right)=O P T\left(\sigma, s^{\prime}, 0\right)$.

If $s^{\prime}=0$, then any packing puts one item of size $L$ into each bin that it uses. By Proposition 6. $O P T\left(\sigma, 2 \ell^{\prime}, 0\right) \geq 2 \ell^{\prime} S$. Since each bin in $\sigma$ has size at most $4 S-3$, it follows that $R\left(\sigma, 0, \ell^{\prime}\right) \leq \ell^{\prime}(4 S-3) \leq O P T\left(\sigma, 2 \ell^{\prime}, 0\right)+\ell^{\prime}(2 S-3)$.

Let $s^{\prime}, \ell^{\prime} \geq 1$ and suppose the claim is true for $s^{\prime \prime}$ and $\ell^{\prime \prime}$, if $0 \leq s^{\prime \prime}<s^{\prime}$ or $0 \leq \ell^{\prime \prime}<\ell^{\prime}$. Let $\sigma$ be any sequence of bins, let $B \leq M=4 S-3$ be the size of the first bin in $\sigma$, and let $\sigma^{\prime}$ be obtained from $\sigma$ by removing its first bin. Since $s^{\prime}, \ell^{\prime} \geq 1$, it follows that $s^{\prime}+2 \ell^{\prime} \geq 3 \geq\lfloor B / S\rfloor$. Note that $O P T\left(\sigma, s^{\prime}+2 \ell^{\prime}, 0\right)=$ $B+O P T\left(\sigma^{\prime}, s^{\prime}+2 \ell^{\prime}-\lfloor B / S\rfloor, 0\right)$, because OPT packs $\lfloor B / S\rfloor$ items of size $S$ in the first bin.

Consider any algorithm. If it packs the first bin with only items of size $S$, then, it packs $\lfloor B / S\rfloor$ items into that bin and the total space it uses is at most $B+R\left(\sigma^{\prime}, s^{\prime}-\right.$ $\left.\lfloor B / S\rfloor, \ell^{\prime}\right)$, which, by the induction hypothesis, is at most $B+O P T\left(\sigma^{\prime}, s^{\prime}+2 \ell^{\prime}-\right.$ $\lfloor B / S\rfloor, 0)+\ell^{\prime}(2 S-3)=O P T\left(\sigma, s^{\prime}+2 \ell^{\prime}, 0\right)+\ell^{\prime}(2 S-3)$. So assume that, in the first bin, the algorithm packs one item of size $L$ plus possibly one item of size $S$.

If $B<L+S$, then the algorithm packs no items of size $S$ into the first bin and uses at most $B+R\left(\sigma^{\prime}, s^{\prime}, \ell^{\prime}-1\right)$ space. By the induction hypothesis, $R\left(\sigma^{\prime}, s^{\prime}, \ell^{\prime}-1\right) \leq$ $O P T\left(\sigma^{\prime}, s^{\prime}+2 \ell^{\prime}-2,0\right)+\left(\ell^{\prime}-1\right)(2 S-3)$. Since OPT packs at most two items of size $S$ into the first bin, $B+O P T\left(\sigma^{\prime}, s^{\prime}+2 \ell^{\prime}-2,0\right) \leq O P T\left(\sigma, s^{\prime}+2 \ell^{\prime}, 0\right)$. Hence, the space used by the algorithm is at most $B+R\left(\sigma^{\prime}, s^{\prime}, \ell^{\prime}-1\right) \leq O P T\left(\sigma, s^{\prime}+2 \ell^{\prime}, 0\right)+\ell^{\prime}(2 S-3)$.

Otherwise, $L+S \leq B \leq M=2 L-1$ and the algorithm also packs one item of size $S$ into the first bin. Then the space used by the algorithm is at most $B+$ $R\left(\sigma^{\prime}, s^{\prime}-1, \ell^{\prime}-1\right) \leq B+O P T\left(\sigma^{\prime}, s^{\prime}-1+2 \ell^{\prime}-2,0\right)+\left(\ell^{\prime}-1\right)(2 S-3)$, by the 
induction hypothesis. Since OPT packs at most three items of size $S$ into the first bin, $B+O P T\left(\sigma^{\prime}, s^{\prime}+2 \ell^{\prime}-3,0\right) \leq O P T\left(\sigma, s^{\prime}+2 \ell^{\prime}, 0\right)$. Hence, the space used by the algorithm is at most $B+R\left(\sigma^{\prime}, s^{\prime}-1, \ell^{\prime}-1\right) \leq O P T\left(\sigma, s^{\prime}+2 \ell^{\prime}, 0\right)+\ell^{\prime}(2 S-3)$.

It follows that, in all cases, $R\left(\sigma, s^{\prime}, \ell^{\prime}\right) \leq O P T\left(\sigma, s^{\prime}+2 \ell^{\prime}, 0\right)+\ell^{\prime}(2 S-3)$.

Now, we consider the case where OPT is the first to run out of something and it runs of items of size $S$. In this case, after this point, the worst sequence for the reasonable packing has bins of size $L$ followed by one bin of size $M$.

Lemma 10. For all sequences of bins $\sigma$ and all integers $s^{\prime}, \ell^{\prime} \geq 0$, if $2 k=s^{\prime}+2 \ell^{\prime}$, then $R\left(\sigma, s^{\prime}, \ell^{\prime}\right) \leq O P T(\sigma, 0, k)+\left(s^{\prime}+\ell^{\prime}-k-1\right) L+M$.

Proof. If $s^{\prime}=0$, then $k=\ell^{\prime}$ and the lemma follows from Proposition 8 So, we assume that $s^{\prime} \geq 1$. Let $\ell^{\prime}$ and $k$ be such that $2 k=s^{\prime}+2 \ell^{\prime}$. Note that $s^{\prime}$ is even, so $s^{\prime} \geq 2$. Let $\sigma$ be a sequence of bins that maximizes $R\left(\sigma, s^{\prime}, \ell^{\prime}\right)-O P T(\sigma, 0, k)$. Suppose that, among all such sequences, $\sigma$ has the smallest capacity (i.e. the smallest sum of bin sizes).

Consider a reasonable packing with $\operatorname{cost} R\left(\sigma, s^{\prime}, \ell^{\prime}\right)$ for $\sigma$. If $\sigma$ has any bins of size in $[S, L-1]$, they are not used by OPT, which has no small items. By the minimality of the capacity of $\sigma$, they are used by the reasonable packing to pack one item of size $S$. All other bins have size at least $L$ and they are used by both the reasonable packing and OPT, unless one of them has already packed all its items.

We begin by making a number of observations about the last bin in $\sigma$, which all follow from the definition of $\sigma$.

The last bin in $\sigma$ must be used by either OPT or the reasonable packing; otherwise, removing it would decrease the capacity of the sequence without changing the total space used by either packing.

If the last bin is only used by the reasonable packing, it must have size $M$; otherwise, replacing it by a bin of size $M$ would increase the total space used by the reasonable packing without changing the total space used by OPT.

If the last bin is only used by OPT, (to pack one item of size $L$ ), it must have size $L$; otherwise, replacing it by a bin of size $L$ would decrease the total space used by OPT without changing the total space used by the reasonable packing.

If the last bin is used by OPT and the reasonable packing to each pack one item, it must have size $L$; otherwise, replacing it by a bin of size $L$ would decrease the capacity of the sequence while changing the total space used by OPT and the reasonable packing by the same amount.

Finally, the last bin cannot be used by the reasonable packing to pack more than one item; otherwise, it must have size between $2 S$ and $M$. If it is replaced by a bin 
of size $L$ followed by a bin of size $M$, the total space used by the reasonable packing would increase, and the space used by OPT would not.

Now suppose that $\sigma$ has a bin, other than its last bin, that does not have size $L$. Let $b$ be the last such bin. Let $s^{\prime \prime}$ and $\ell^{\prime \prime}$ be the number of items of size $S$ and $L$, respectively, that the reasonable packing has remaining immediately after packing bin $b$. These items are packed one per bin in each of the next $s^{\prime \prime}+\ell^{\prime \prime}$ bins. Since the packing is reasonable, the items of size $L$ are packed before the items of size $S$. If $k^{\prime \prime}$ is the number of items (of size $L$ ) that OPT has remaining immediately after bin $b$, they are packed one per bin in each of the next $k^{\prime \prime}$ bins.

If size $(b) \leq L-1$, then the reasonable packing packs a single item of size $S$ in this bin. Increasing the size of $b$ to $L$ does not change which bins the reasonable packing uses: it still packs one item in each of the $1+s^{\prime \prime}+\ell^{\prime \prime}$ bins starting with $b$, since all of them, except possibly the last, have size $L$. This increases the total space used by the reasonable packing. However, the total space used by OPT remains unchanged. This is because, if OPT has any items remaining immediately before bin $b$ is packed, they are each packed in a bin of size $L$. This contradicts the definition of $\sigma$. Since size $(b) \neq L$, it follows that size $(b)>L$.

If bin $b$ is only used by OPT (to pack one item of size $L$ ), then replacing it by a bin of size $L$ would decrease the total space used by OPT without changing the total space used by the reasonable packing, contradicting the definition of $\sigma$. Therefore the reasonable packing packs at least one item in bin $b$.

Suppose that OPT uses bin $b$. If the reasonable packing packs one item in bin $b$, then decreasing the size of bin $b$ to $L$ would decrease the capacity of the sequence while changing the total cost incurred by OPT and the reasonable packing by the same amount, contradicting the definition of $\sigma$. Hence the reasonable packing packs $h>1$ items in bin $b$. Decreasing the size of bin $b$ to $L$ and adding $h-1$ bins of size $L$ immediately following bin $b$ decreases the total space used by OPT by size $(b)-L>0$, since OPT still packs each item following bin $b$ in a bin of size $L$. It also increases the total space used by the reasonable packing by $h L-\operatorname{size}(b)>0$, since it packs one item in each bin, from bin $b$ onward. This contradicts the definition of $\sigma$. Therefore, OPT does not use bin $b$. Since size $(b)>L$, OPT runs out of items before bin $b$.

We now show that, at the point OPT runs out of items, the reasonable packing only has items of size $S$.

Consider the last bin $b^{\prime}$ in $\sigma$ prior to which OPT has at least one item (of size $L$ ) available and the reasonable packing has items of both size $S$ and size $L$ available. Then, up to including when bin $b^{\prime}$ is packed, we claim that twice the number of items of size $L$ that OPT has available is at least the number of items of size $S$ plus twice the number of items of size $L$ that the reasonable packing has available. In particular, it is true before the first bin of $\sigma$ is packed, since $2 k=s^{\prime}+2 \ell^{\prime}$. For each 
bin of size at least $L$, up to and including bin $b^{\prime}$, OPT packs one item of size $L$ and the reasonable algorithm packs at least one item of size $L$ or at least two items of size $S$, so the inequality remains true. Moreover, OPT packs no items in bins whose size is less than $L$, so such bins do not cause the inequality to become false.

It follows that if OPT runs out of items immediately after bin $b^{\prime}$, then the reasonable packing does too. Since OPT does not use bin $b$, but the reasonable packing does, OPT runs out of items prior to the reasonable packing, so this is impossible. If the reasonable packing runs out of items of size $S$ immediately after bin $b^{\prime}$, then OPT has at least as many items of size $L$ left as the reasonable packing and, hence, runs out of items at or after the reasonable packing, so there is a contradiction again.

Therefore, immediately after bin $b^{\prime}$ and, hence, immediately before bin $b$, the reasonable packing only has items of size $S$ left. Since bin $b$ is not the last bin, the reasonable packing is feasible, and size $(b)>L$, the reasonable packing has at least two items in $b$. Replace bin $b$ by a bin of size size $(b)-S \geq S$ followed by a bin of size $L$. Then the reasonable packing must pack one fewer item of size $S$ in the first of these bins and one item of size $S$ in the second. This adds $L-S$ to the the total space used by the reasonable packing, but decreases the total space used by OPT, contradicting the definition of $\sigma$. Therefore, bin $b$ does not exist.

Thus, we may assume that $\sigma$ consists of $s^{\prime}+\ell^{\prime}-1 \geq k$ bins of size $L$, followed by at most one bin of size $M$. Then $O P T(\sigma, 0, k)=k L$ and $R\left(\sigma, s^{\prime}, \ell^{\prime}\right) \leq\left(s^{\prime}+\ell^{\prime}-1\right) L+M$, so $R\left(\sigma, s^{\prime}, \ell^{\prime}\right)-O P T(\sigma, 0, k) \leq\left(s^{\prime}+\ell^{\prime}-k-1\right) L+M$.

Next, we consider the case where $R$ is the first to run out of something and it runs of items of size $L$.

Lemma 11. For all sequences of bins $\sigma$ and all integers $s^{\prime}, \ell^{\prime} \geq 0$, if $k \geq s^{\prime}+2 \ell^{\prime}$, then $R(\sigma, k, 0) \leq O P T\left(\sigma, s^{\prime}, \ell^{\prime}\right)+\left(k-s^{\prime}-\ell^{\prime}-1\right) L+M$.

Proof. by induction on $s^{\prime}$ and $\ell^{\prime}$.

If $\ell^{\prime}=0$, then the first $s^{\prime}$ items of a reasonable packing are packed the same as OPT would pack them. Since the packing is feasible, each bin that contains one of the remaining $k-s^{\prime}$ items of size $S$ has at most $S-1$ empty space, except for the last such bin, which has at most $M-S$ empty space. Thus $R(\sigma, k, 0) \leq$ $\operatorname{OPT}\left(\sigma, s^{\prime}, 0\right)+\left(k-s^{\prime}\right) S+\left(k-s^{\prime}-1\right)(S-1)+M-S=\mathrm{OPT}\left(\sigma, s^{\prime}, 0\right)+\left(k-s^{\prime}-1\right) L+M$.

Next consider $s^{\prime}=0$. Any packing of $k$ items of size $S$ has at most $S-1$ empty space in each bin that it uses, except the last, which has at most $M-S$ empty space. Since it uses at most $k$ bins, its cost is at most $k S+(k-1)(S-1)+M-S=(k-1) L+M$. By Proposition 6. OPT $\left(\sigma, 0, \ell^{\prime}\right) \geq \ell^{\prime} L$, so $R(\sigma, k, 0) \leq(k-1) L+M=\ell^{\prime} L+(k-$ $\left.\ell^{\prime}-1\right) L+M \leq \operatorname{OPT}\left(\sigma, 0, \ell^{\prime}\right)+\left(k-\ell^{\prime}-1\right) L+M$. 
Now, let $s^{\prime}, \ell^{\prime}>0$ and suppose the claim is true if $s^{\prime \prime}<s^{\prime}$ or $\ell^{\prime \prime}<\ell^{\prime}$. Let $\sigma$ be any sequence of bins, and let $B \leq M=4 S-3$ be the size of its first bin. Since $k \geq s^{\prime}+2 \ell^{\prime} \geq 3$, any reasonable packing of $k$ items of size $S$ puts $\lfloor B / S\rfloor \leq 3$ items into this bin, so has cost at most $B+R\left(\sigma^{\prime}, k-\lfloor B / S\rfloor, 0\right)$, where $\sigma^{\prime}$ is the sequence $\sigma$ without its first bin.

If OPT packs the first bin with only items of size $S$, then, it packs $\lfloor B / S\rfloor \leq s^{\prime}$ items into that bin and the total space it uses is $B+\operatorname{OPT}\left(\sigma^{\prime}, s^{\prime}-\lfloor B / S\rfloor, \ell^{\prime}\right)$. By the induction hypothesis, $B+R\left(\sigma^{\prime}, k-\lfloor B / S\rfloor, 0\right) \leq B+\mathrm{OPT}\left(\sigma^{\prime}, s^{\prime}-\lfloor B / S\rfloor, \ell^{\prime}\right)+$ $\left(k-\lfloor B / S\rfloor-\left(s^{\prime}-\lfloor B / S\rfloor\right)-\ell^{\prime}-1\right) L+M=\operatorname{OPT}\left(\sigma, s^{\prime}, \ell^{\prime}\right)+\left(k-s^{\prime}-\ell^{\prime}-1\right) L+M$.

Otherwise OPT packs the first bin with one item of size $L$ and $\lfloor(B-L) / S\rfloor \geq$ $\lfloor B / S\rfloor-2$ items of size $S$. Since $k-\lfloor B / S\rfloor \geq s^{\prime}+2 \ell^{\prime}-\lfloor(B-L) / S\rfloor-2=$ $\left(s^{\prime}-\lfloor(B-L) / S\rfloor\right)+2\left(\ell^{\prime}-1\right)$, it follows by the induction hypothesis that $B+$ $R\left(\sigma^{\prime}, k-\lfloor B / S\rfloor, 0\right) \leq B+\operatorname{OPT}\left(\sigma^{\prime}, s^{\prime}-\lfloor(B-L) / S\rfloor, \ell^{\prime}-1\right)+\left(k-\lfloor B / S\rfloor-\left(s^{\prime}-\right.\right.$ $\left.\lfloor B / S-2\rfloor)-\left(\ell^{\prime}-1\right)-1\right) L+M \leq \operatorname{OPT}\left(\sigma, s^{\prime}, \ell^{\prime}\right)+\left(k-s^{\prime}-\ell^{\prime}-1\right) L+M$. Note that this last case is the only place that the possibility of $k$ being greater than $s^{\prime}+2 \ell^{\prime}$ is used.

Hence, $R(\sigma, k, 0) \leq B+R\left(\sigma^{\prime}, k-\lfloor B / S\rfloor, 0\right) \leq \mathrm{OPT}\left(\sigma, s^{\prime}, \ell^{\prime}\right)+\left(k-s^{\prime}-\ell^{\prime}-1\right) L+M \square$

Finally, we consider the case where $R$ is the first to run out of something and it runs of items of size $S$.

Lemma 12. For all sequences of bins $\sigma$ and all integers $s^{\prime}, \ell^{\prime} \geq 0$, if $2 k \geq s^{\prime}+2 \ell^{\prime}$, then $R(\sigma, 0, k) \leq \operatorname{OPT}\left(\sigma, s^{\prime}, \ell^{\prime}\right)+\min \left\{0, \ell^{\prime}-s^{\prime}\right\} S+\left(k-\ell^{\prime}\right) M$.

Proof. by induction on $s^{\prime}$ and $\ell^{\prime}$.

If $s^{\prime}=0$, then the first $\ell^{\prime}$ items are packed the same way in a reasonable packing as OPT would pack them. Each of the remaining $k-\ell^{\prime}$ items of size $L$ are packed one per bin using bins of size at most $M$, for a total cost at most $\operatorname{OPT}\left(\sigma, 0, \ell^{\prime}\right)+$ $\left(k-\ell^{\prime}\right) M=\operatorname{OPT}\left(\sigma, 0, \ell^{\prime}\right)+\min \left\{0, \ell^{\prime}-s^{\prime}\right\} S+\left(k-\ell^{\prime}\right) M$, since $\ell^{\prime}-s^{\prime} \geq 0$.

If $\ell^{\prime}=0$, then, by Proposition 6 . OPT $\left(\sigma, s^{\prime}, \ell^{\prime}\right) \geq s^{\prime} S+\ell^{\prime} L=\max \left\{0, s^{\prime}-\ell^{\prime}\right\} S$, so $\operatorname{OPT}\left(\sigma, s^{\prime}, \ell^{\prime}\right)+\min \left\{0, \ell^{\prime}-s^{\prime}\right\} S \geq 0$. In a reasonable packing, each of the $k$ items of size $L$ are packed one per bin using bins of size at most $M$ for a total cost at most $k M \leq \operatorname{OPT}\left(\sigma, s^{\prime}, \ell^{\prime}\right)+\min \left\{0, \ell^{\prime}-s^{\prime}\right\} S+\left(k-\ell^{\prime}\right) M$.

Now, let $s^{\prime}, \ell^{\prime} \geq 1$ and suppose the claim is true if $s^{\prime \prime}<s^{\prime}$ or $\ell^{\prime \prime}<\ell^{\prime}$. Let $\sigma$ be any sequence of bins, let $B \leq M=4 S-3$ be the size of its first bin, and let $\sigma^{\prime}$ denote the same sequence as $\sigma$, but with the first bin removed.

If $B<L$, then a reasonable packing leaves the first bin of $\sigma$ empty, since it has no items of size $S$. Hence, it uses space at most $R\left(\sigma^{\prime}, 0, k\right)$. Since $2 k \geq s^{\prime}+2 \ell^{\prime} \geq$ $\left(s^{\prime}-1\right)+2 \ell^{\prime}$, the induction hypothesis implies that $R\left(\sigma^{\prime}, 0, k\right) \leq \operatorname{OPT}\left(\sigma^{\prime}, s^{\prime}-1, \ell^{\prime}\right)+$ 
$\min \left\{0, \ell^{\prime}-\left(s^{\prime}-1\right)\right\} S+\left(k-\ell^{\prime}\right) M$. OPT packs one item of size $S$ into the first bin of $\sigma$, so $\operatorname{OPT}\left(\sigma, s^{\prime}, \ell^{\prime}\right)=B+\operatorname{OPT}\left(\sigma^{\prime}, s^{\prime}-1, \ell^{\prime}\right)$. Thus, since $S \leq B$, it follows that $R\left(\sigma^{\prime}, 0, k\right) \leq \operatorname{OPT}\left(\sigma^{\prime}, s^{\prime}-1, \ell^{\prime}\right)+B-S+\min \left\{0, \ell^{\prime}-\left(s^{\prime}-1\right)\right\} S+\left(k-\ell^{\prime}\right) M \leq$ $\operatorname{OPT}\left(\sigma, s^{\prime}, \ell^{\prime}\right)+\min \left\{0, \ell^{\prime}-s^{\prime}\right\} S+\left(k-\ell^{\prime}\right) M$.

Otherwise, $B \geq L$. Then, after packing the first bin of $\sigma$, a reasonable packing has $k-1$ items (of size $L$ ) remaining. Suppose OPT has $s^{\prime \prime}$ items of size $S$ and $\ell^{\prime \prime}$ items of size $L$ remaining after packing this bin. Hence, by the induction hypothesis, to pack its $k-1$ items of size $L$, any reasonable packing uses space at most $R\left(\sigma^{\prime}, 0, k-1\right) \leq$ $\operatorname{OPT}\left(\sigma^{\prime}, s^{\prime \prime}, \ell^{\prime \prime}\right)+\min \left\{0, \ell^{\prime \prime}-s^{\prime \prime}\right\} S+\left(k-1-\ell^{\prime \prime}\right) M$, since $2(k-1) \geq s^{\prime}+2\left(\ell^{\prime}-1\right)=\left(s^{\prime}-\right.$ $2)+2 \ell^{\prime} \geq s^{\prime \prime}+2 \ell^{\prime \prime}$. Therefore, $R(\sigma, 0, k)=B+R\left(\sigma^{\prime}, 0, k-1\right) \leq B+\mathrm{OPT}\left(\sigma^{\prime}, s^{\prime \prime}, \ell^{\prime \prime}\right)+$ $\min \left\{0, \ell^{\prime \prime}-s^{\prime \prime}\right\} S+\left(k-1-\ell^{\prime \prime}\right) M=\mathrm{OPT}\left(\sigma, s^{\prime}, \ell^{\prime}\right)+\min \left\{0, \ell^{\prime \prime}-s^{\prime \prime}\right\} S+\left(k-1-\ell^{\prime \prime}\right) M$.

It remains to be shown that

$$
\min \left\{0, \ell^{\prime \prime}-s^{\prime \prime}\right\} S+\left(k-1-\ell^{\prime \prime}\right) M \leq \min \left\{0, \ell^{\prime}-s^{\prime}\right\} S+\left(k-\ell^{\prime}\right) M .
$$

Since OPT is reasonable and $B \leq M=2 L-1=4 S-3$, either $\ell^{\prime \prime}=\ell^{\prime}-1$ and $s^{\prime \prime} \geq s^{\prime}-1$ or $\ell^{\prime \prime}=\ell^{\prime}$ and $s^{\prime \prime} \geq s^{\prime}-3$. In the first case, 1 follows since $\ell^{\prime \prime}-s^{\prime \prime} \leq$ $\left(\ell^{\prime}-1\right)-\left(s^{\prime}-1\right)=\ell^{\prime}-s^{\prime}$ and $k-1-\ell^{\prime \prime}=k-\ell^{\prime}$, so $\min \left\{0, \ell^{\prime \prime}-s^{\prime \prime}\right\} S+\left(k-1-\ell^{\prime \prime}\right) M \leq$ $\min \left\{0, \ell^{\prime}-s^{\prime}\right\} S+\left(k-\ell^{\prime}\right) M$. In the second case, 1 follows since $\ell^{\prime \prime}-s^{\prime \prime} \leq \ell^{\prime}-s^{\prime}+3$ and $k-1-\ell^{\prime \prime}=k-\ell^{\prime}-1$, so $\min \left\{0, \ell^{\prime \prime}-s^{\prime \prime}\right\} S+\left(k-1-\ell^{\prime \prime}\right) M \leq \min \left\{0, \ell^{\prime}-s^{\prime}\right\} S+$ $3 S+\left(k-\ell^{\prime}\right) M-M \leq \min \left\{0, \ell^{\prime}-s^{\prime}\right\} S+\left(k-\ell^{\prime}\right) M$, since $3 S \leq 4 S-3=M$, provided $S \geq 3$. Thus, asymptotically (for $S \geq 3), R(\sigma, 0, k) \leq \mathrm{OPT}\left(\sigma, s^{\prime}, \ell^{\prime}\right)+\min \left\{0, \ell^{\prime \prime}-\right.$ $\left.s^{\prime \prime}\right\} S+\left(k-1-\ell^{\prime \prime}\right) M \leq \operatorname{OPT}\left(\sigma, s^{\prime}, \ell^{\prime}\right)+\min \left\{0, \ell^{\prime}-s^{\prime}\right\} S+\left(k-\ell^{\prime}\right) M$.

\section{Simple Non-Optimal Algorithms}

It is helpful to understand why simple reasonable algorithms are not optimal for the Restricted Grid Scheduling problem. The following examples show why a number of natural candidates do not work well enough.

Example 1 Consider the reasonable algorithm that always uses items of size $S$, when there is a choice. Let $s=2 \ell$ and let $\sigma$ consist of $\ell$ bins of size $2 S$, followed by $s$ bins of size $S$, and then $\ell$ bins of size $M$. For this instance, the algorithm has a performance ratio of $\frac{\ell \cdot 2 S+\ell \cdot M}{\ell \cdot 2 S+s \cdot S}=\frac{3}{2}-\frac{3}{4 S}$, which is greater than $5 / 4$ for large $S$.

Example 2 Consider the reasonable algorithm that always uses items of size $L$, when there is a choice. Let $s=2 \ell$ and let $\sigma$ consist of $\ell$ bins of size $2 S$, followed by $s-1$ bins of size $L$ and then one bin of size $M$. For this instance, the algorithm has a performance ratio of $\frac{\ell \cdot 2 S+(s-1) \cdot L+M}{\ell \cdot 2 S+\ell \cdot L}=\frac{3}{2}+\frac{1}{2 \ell}-\frac{3 / \ell+1}{2(4 S-1)}$, which is also greater than $5 / 4$ for large $S$.

For the two instances considered above, the reasonable algorithm which alternates between using two items of size $S$ and one item of size $L$, when it has a choice, 
would do well, achieving a performance ratio of $5 / 4$. However, it does not do as well on other instances.

Example 3 Let $2 s=3 \ell$ and let $\sigma$ consist of $\ell$ bins of size $2 S$, followed by $s$ bins of size $S$ and $\ell / 2$ bins of size $M$. For this instance, the algorithm which alternates between using two items of size $S$ and one item of size $L$, when it has a choice, has a performance ratio of $\frac{\ell \cdot 2 S+(s-\ell) \cdot S+\ell / 2 \cdot M}{\ell \cdot 2 S+s \cdot S}=1+\frac{\frac{\ell}{2} \cdot(M-2 S)}{(2 \ell+s) \cdot S}=1+\frac{2}{7}-\frac{3}{7 S}$, which is larger than $5 / 4$ for $S$ sufficiently large.

As the above examples partially illustrate, once either the online algorithm or OPT has run out of one type of item (items of size $S$ or items of size $L$ ), the adversary can give bins which make the online algorithm waste a lot of space. The algorithm we present in the next section aims to postpone this situation long enough to get a good ratio.

When $3 s=2 \ell$, the lower bound is in Case II. It starts by giving the algorithm $\lfloor s / 2\rfloor$ bins of size $2 S$ and it considers the number $k$ of these bins in which the algorithm packs two items of size $S$. The best the algorithm can do is to have $k \approx \max \{\lfloor s / 2\rfloor-s / 8-\ell / 4+1,\lfloor s / 2\rfloor-s / 3+1\}=\lfloor s / 2\rfloor-s / 3+1 \approx s / 6$, so that the same ratio is obtained for both subcases. This is the same as using one item of size $L$ twice as often as two items of size $S$, when it has a choice.

Example 4 Let $3 s=2 \ell$. Consider the reasonable algorithm which uses one item of size $L$ twice as often as two items of size $S$, when it has a choice. Let $\sigma$ consist of $\ell$ bins of size $2 S$, followed by $s$ bins of size $S$ and $\ell / 3$ bins of size $M$. For this instance, the algorithm packs $\ell / 3=s / 2$ bins of size $2 S$ with two items of size $S$, so it has a performance ratio of $\frac{\ell \cdot 2 S+(\ell / 3) \cdot M}{\ell \cdot 2 S+s \cdot S}=1+\frac{\frac{s}{2} \cdot M-s S}{(2 \ell+s) \cdot S}=1+\frac{S-3 / 2}{4 \cdot S}=\frac{5}{4}-\frac{3}{8 S}$, which exceeds the lower bound of $1+\frac{2}{3+6 \ell / s}=\frac{7}{6}$ for $S$ sufficiently large.

This example shows that it is not always possible to achieve the lower bound by choosing to use two items of size $S$ instead of one item of size $L$ a fixed proportion of the time. After $s / 6$ bins have been packed with two items of size $S$, it turns out to be better to always use an item of size $L$, when there is a choice. This motivates our use of a second phase to obtain the best competitive ratio. The number of items of size $S$ that the algorithm has packed, in this case $s / 6 \times 2=s / 3$, indicates when the first phase is done.

\section{2-Phase-Packer}

In Figure 2, we present a reasonable algorithm, 2-Phase-Packer, for the Restricted Grid Scheduling problem. It is asymptotically optimal: the competitive ratio matches the lower bound in Section 3 for all three ranges of the ratio $s / \ell$ of the initial numbers of items of size $S$ and items of size $L$. Not surprisingly, 2-Phase-Packer has two phases. 
In the first phase, it attempts to balance the number of items of size $S$ and the number of items of size $L$ it uses, aiming for the ratio indicated by the lower bound. When it receives bins where it has a choice of using one item of size $L$ or two items of size $S$ in part or all of that bin (i.e., bins with sizes in the ranges $[2 S, 3 S-2]$ and $[3 S, 4 S-3]$ ), it uses one item of size $L$ in a certain fraction of them (and increments the variable L-bins) and uses two items of size $S$ in the remaining fraction (and increments S-bins). The fraction varies depending on the original ratio $s / \ell$ : at least 2 , between 2 and $6 / 5$, and less than $6 / 5$. It is enforced by a macro called UseLs, which indicates that an item of size $L$ should be packed if and only if L-bins $\leq r \mathrm{~S}$-bins, where $r$ is the target ratio of L-bins to S-bins. For example, when the number of bins containing one item of size $L$ should be within 1 of the number of bins containing two items of size $S$, we have UseLs $=$ (L-bins $\leq$ S-bins). Both L-bins and S-bins start at zero, so, in this case, 2-Phase-Packer starts by choosing to use one item of size $L$ and then alternates. Note that S-bins and L-bins do not change after Phase 1 is completed.

In the middle range for the ratio $s / \ell$, there are two different fractions used. The first fraction is used until S-bins reaches a specific value. Afterwards, its choices alternate. To do so, for the rest of Phase 1, it records the number of times it chooses to pack two items of size $S$ in a bin and the number of times it chooses to pack one item of size $L$ in late-S-bins and late-L-bins, respectively. The variables late-S-bins and late-L-bins are also zero initially.

2-Phase-Packer uses countS and countL throughout the algorithm to keep track of the total numbers of items of size $S$ and items of size $L$ it has packed, whether or not it had a choice. (Specifically, countS is incremented every time an item of size $S$ is packed and countL is incremented every time an item of size $L$ is packed.) It continues with Phase 1 until it has packed a certain number of items of size $S$ or items of size $L$ (depending on the relationship between $s$ and $\ell$ ). For each of the three ranges of $s / \ell$, we define a different condition for ending Phase 1. In Phase 2, only items of size $S$ or only items of size $L$ are packed where a reasonable algorithm has a choice, depending on whether one would expect an excess of items of size $S$ or items of size $L$, given the ratio $s / \ell$.

An example of an execution of 2-Phase-Packer Consider running 2-Phase-Packer when $\ell=s / 2$ is even and the sequence of bins consists of $3 \ell / 2$ bins of size $2 S$, followed by $\ell$ bins of size $S$, and then $\ell / 2$ bins of size $M$. During Phase 1, 2-Phase-Packer alternates between packing an item of size $L$ and two items of size $S$, starting with an item of size $L$, because the condition (L-bins $\leq$ S-bins) will alternate between being true and false. Phase 1 ends when $\ell / 2$ items of size $L$ have been packed. In Phase 2, the remaining $\ell / 2$ items of size $L$ and $s / 2+2$ items of size $S$ are packed. These items of size $S$ are packed in the last $\ell / 2+1$ bins of size $2 S$. 2-Phase-Packer leaves the bins of size $S$ empty, because no items of size $S$ remain. It puts one item of size $L$ in each bin of size $M$. Note that 2-Phase-Packer 
has cost $(3 \ell / 2) \cdot 2 S+(\ell / 2) \cdot M=5 \ell S-3 \ell / 2$. In contrast, OPT packs all the items of size $L$ in bins of size $2 S$, packs half the items of size $S$ in bins of size $2 S$, and packs the other half in the bins of size $S$. Its cost is $3 \ell / 2 \cdot 2 S+\ell S=4 \ell S$. The ratio of their costs is $\frac{5 \ell S-\frac{3 \ell}{2}}{4 \ell S}$.

The definition of the algorithm implies the following relationships.

Observation 1. L-bins + late-L-bins $\leq$ countL and $2($ S-bins + late-S-bins $) \leq$ countS.

If $\ell \leq s / 2$ or $5 s / 6<\ell$, then late-S-bins $=$ late-L-bins $=0$.

The definitions of the end of Phase 1 for the various ranges of $s / \ell$ imply these inequalities.

Lemma 13. If $s / 2<\ell \leq 5 s / 6$, then $S$-bins + late-S-bins $\leq 3 s / 8-\ell / 4+1$.

If $5 s / 6<\ell$, then $S$-bins $\leq s / 6+1$.

The following simple invariants can be proved inductively.

Lemma 14. If $\ell \leq s / 2$, then $S$-bins $\leq L$-bins $\leq S$-bins +1 .

If $s / 2<\ell \leq 5 s / 6$, then late-S-bins $\leq$ late-L-bins $\leq$ late-S-bins +1 and

$\lfloor c(S$-bins -1$)\rfloor+1 \leq L$-bins $\leq\lfloor c$-bins $\rfloor+1$, where $1<c=\frac{10 \ell-3 s}{s+2 \ell} \leq 2$.

If $5 s / 6<\ell$, then $2 S$-bins $\leq L$-bins $\leq 2 S$-bins +1 .

We are now ready to prove the following:

Theorem 15. The Restricted Grid Scheduling problem has competitive ratio at most

$$
\begin{cases}1+\frac{1}{2+s / \ell} & \text { if } \ell \leq s / 2 \\ 1+\frac{1}{4} & \text { if } s / 2<\ell \leq 5 s / 6, \text { and } \\ 1+\frac{2}{3+6 \ell / s} & \text { if } 5 s / 6<\ell\end{cases}
$$

Proof. We analyse the performance of 2-Phase-Packer as compared to the cost of an optimal reasonable packing on an arbitrary sequence, $\sigma$, using the three different ranges for the relationship between $s$ and $\ell$ in the lower bound.

Both 2-Phase-Packer and OPT use exactly the same bins until one of them runs out of either items of size $L$ or items of size $S$. Thus, there are four cases to consider. 
macro Phase1done $= \begin{cases}\operatorname{count} \mathrm{L} \geq\lfloor\ell / 2\rfloor & \text { if } \ell \leq s / 2 \\ \operatorname{count} \mathrm{S} \geq\lfloor 3 s / 4-\ell / 2\rfloor & \text { if } s / 2<\ell \leq 5 s / 6 \\ \operatorname{countS} \geq\lfloor s / 3\rfloor & \text { if } 5 s / 6<\ell\end{cases}$

macro UseLs $= \begin{cases}\text { L-bins } \leq \mathrm{S}-\text { bins } & \text { if } \ell \leq s / 2 \\ \text { if }(\mathrm{S}-\text { bins }<\lfloor(s+2 \ell) / 16\rfloor) & \text { if } s / 2<\ell \leq 5 s / 6 \\ \text { then L-bins } \leq(10 \ell-3 s) \text { S-bins } /(s+2 \ell) & \\ \text { else late-L-bins } \leq \text { late-S-bins } & \\ \text { L-bins } \leq 2 \mathrm{~S}-\text { bins } & \text { if } 5 s / 6<\ell\end{cases}$

countS \% counts the number of items of size $S$ packed; initially 0 countL $\%$ counts the number of items of size $L$ packed; initially 0

S-bins $\leftarrow$ L-bins $\leftarrow$ late-S-bins $\leftarrow$ late-L-bins $\leftarrow 0$

for each arriving bin $b$

if only one type of item still remains then use as many items as fit in $b$

else if size $(b) \in[S, 2 S-2]$ then use 1 item of size $S$

else if size $(b)=2 S-1$ then use 1 item of size $L$

else if size $(b)=3 S-1$ then use 1 item of size $L$ and 1 item of size $S$

else if only one item of size $S$ still remains

then use 1 item of size $L$

if remaining space in $b$ is at least $S$ then use 1 item of size $S$

else if only two items of size $S$ still remain and size $(b) \in[3 S, 4 S-3]$

then use 1 item of size $L$ and 1 item of size $S$

else if (not Phase1done)

then \% Use range determined ratio

$$
\text { if } \operatorname{size}(b) \in[2 S, 3 S-2] \text { then }
$$

if UseLs then use 1 item of size $L$

else use 2 items of size $S$

else $\%$ size $(b) \in[3 S, 4 S-3]$

if UseLs then use 1 item of size $L$ and 1 item of size $S$

else use 3 items of size $S$

if $s / 2<\ell \leq 5 s / 6$ and S-bins $\geq\lfloor(s+2 \ell) / 16\rfloor$ then

if UseLs then late-L-bins ++

else late-S-bins ++

else $\% \ell \leq s / 2$ or $\ell>5 s / 6$ or S-bins $<\lfloor(s+2 \ell) / 16\rfloor$

if UseLs then L-bins ++

else S-bins ++

else \% In Phase 2

if $\ell \leq s / 2$ then use as many items of size $S$ as fit in bin $b$

else use 1 item of size $L$

if remaining space in $b$ is at least $S$ then use 1 item of size $S$

end for

Fig. 2. The algorithm 2-Phase-Packer 
(1) OPT runs out of items of size $L$ at or before the point where 2-Phase-Packer runs out of anything.

(2) OPT runs out of items of size $S$ at or before the point where 2-Phase-Packer runs out of anything.

(3) 2-Phase-Packer runs out of items of size $L$ before OPT runs out of anything.

(4) 2-Phase-Packer runs out of items of size $S$ before OPT runs out of anything.

We examine each of these four cases and, within each case, we consider three different ranges for the relationship between $s$ and $\ell$.

Consider an arbitrary sequence of bins $\sigma$ in which 2-Phase-Packer packs $s$ items of size $S$ and $\ell$ items of size $L$. Consider the first bin $b^{\prime}$ after which either OPT or 2-Phase-Packer had only one type of item remaining. Up to and including bin $b^{\prime}$, both OPT and 2-Phase-Packer have unpacked items of both sizes. Let countL' and count $S^{\prime}$ denote the number of items of size $L$ and items of size $S$, respectively, that 2-Phase-Packer has packed up to and including bin $b^{\prime}$. Let S-bins' and late-S-bins' be the values of S-bins and late-S-bins immediately after bin $b^{\prime}$, so S-bins' ${ }^{\prime}+$ late-S-bins' denotes the number of bins at or before $b^{\prime}$ where 2-Phase-Packer had a choice and packed two items of size $S$ instead of one of size $L$. Similarly, L-bins ${ }^{\prime}+$ late-L-bins' denotes the number in which 2-Phase-Packer packed one item of size $L$ instead of two items of size $S$, where L-bins' and late-L-bins' are the values of L-bins and late-L-bins immediately after bin $b^{\prime}$. Let $\sigma^{\prime}$ denote the portion of $\sigma$ after bin $b^{\prime}$.

Both algorithms use all bins up to and including bin $b^{\prime}$, since all bins have size at least $S$. Let $X$ be the total cost of these bins. Then $X \geq\left(\operatorname{count} \mathrm{S}^{\prime}\right) S+\left(\operatorname{countL}^{\prime}\right) L$ and 2-Phase-Packer $(\sigma, s, \ell)=X+2$-Phase-Packer $\left(\sigma^{\prime}, s-\operatorname{countS}^{\prime}, \ell-\operatorname{countL}^{\prime}\right)$.

Case 1: OPT has no items of size $\boldsymbol{L}$ after $\boldsymbol{b}^{\prime}$ In this case, Lemma 5 implies that, after bin $b^{\prime}$, OPT has $\left(s-\right.$ countS $\left.S^{\prime}\right)+2\left(\ell-\right.$ countL $\left.L^{\prime}\right)$ items of size $S$ remaining, so $\operatorname{OPT}(\sigma, s, \ell)=X+O P T\left(\sigma^{\prime},\left(s-\operatorname{count}^{\prime}\right)+2\left(\ell-\operatorname{countL}^{\prime}\right), 0\right)$.

Since 2-Phase-Packer is reasonable, Lemma 9 implies that 2-Phase-Packer $\left(\sigma^{\prime}, s-\right.$ count $\mathrm{S}^{\prime}, \ell-$ countL $\left.^{\prime}\right) \leq \mathrm{OPT}\left(\sigma^{\prime},\left(s-\operatorname{count}^{\prime}\right)+2\left(\ell-\operatorname{count}^{\prime}\right), 0\right)+\left(\ell-\operatorname{countL}^{\prime}\right)(2 S-$ $3)$, so

$$
\text { 2-Phase-Packer }(\sigma, s, \ell) \leq \operatorname{OPT}(\sigma, s, \ell)+\left(\ell-\operatorname{count}^{\prime}\right)(2 S-3) .
$$

By Proposition 6. $\operatorname{OPT}(\sigma, s, \ell) \geq s S+\ell L \geq s L / 2+\ell L=(s+2 \ell) L / 2$. When computing the competitive ratio, we will choose the additive constant to be at least $L$, so we subtract $L$ from 2-Phase-Packer's cost in the ratio. Thus,

$$
\begin{aligned}
\frac{2-\mathrm{Phase}-\mathrm{Packer}(\sigma, s, \ell)-L}{\operatorname{OPT}(\sigma, s, \ell)} & \leq \frac{\mathrm{OPT}(\sigma, s, \ell)+\left(\ell-\operatorname{countL}^{\prime}\right)(2 S-3)-L}{\operatorname{OPT}(\sigma, s, \ell)} \\
& \leq 1+\frac{\left(\ell-\operatorname{countL}^{\prime}-1\right)(L-2)}{(s+2 \ell) L / 2} \\
& \leq 1+\frac{2\left(\ell-\operatorname{countL}^{\prime}-1\right)}{s+2 \ell} .
\end{aligned}
$$


It remains to bound $\ell-$ countL $^{\prime}-1$ in each of the three ranges for the ratio $s / \ell$. We use the fact that, immediately after bin $b^{\prime}$, OPT has run out of items of size $L$, so at least $\ell$ bins of size at least $L$ are in $\sigma$ up to and including bin $b^{\prime}$.

First, consider the case when $\ell \leq s / 2$. If countL' $<\lfloor\ell / 2\rfloor$, then Phase 1 was not completed when bin $b^{\prime}$ arrived. Therefore, each time a bin of size at least $L$ arrives up to and including bin $b^{\prime}, 2$-Phase-Packer either packs an item of size $L$ in it and, hence, increments countL, or it increments S-bins. Hence, countL' $\mathrm{L}^{\prime} \mathrm{S}$-bins ${ }^{\prime} \geq \ell$. By Lemma 14, L-bins' $\geq$ S-bins'. Since countL' $\geq$ L-bins', by Observation 11 it follows that countL' $\geq \ell / 2 \geq\lfloor\ell / 2\rfloor$. This is a contradiction. Thus, countL' $\geq\lfloor\ell / 2\rfloor$ and

$$
\begin{aligned}
\frac{2-\mathrm{Phase}-\mathrm{Packer}(\sigma, s, \ell)-L}{\operatorname{OPT}(\sigma, s, \ell)} & \leq 1+\frac{2\left(\ell-\operatorname{countL}^{\prime}-1\right)}{s+2 \ell} \\
& \leq 1+\frac{\ell}{s+2 \ell}=1+\frac{1}{2+s / \ell} .
\end{aligned}
$$

So, suppose that $s / 2<\ell$. During Phase 1 , each time a bin of size at least $L$ arrives, 2-Phase-Packer either packs an item of size $L$ in it and, hence, increments countL, or it increments one of S-bins or late-S-bins. During Phase 2, 2-Phase-Packer packs an item of size $L$ in each such bin until it runs out of items of size $L$. Therefore, countL $^{\prime}+$ S-bins $^{\prime}+$ late-S-bins ${ }^{\prime} \geq \ell$.

If $\ell \leq 5 s / 6$, then Lemma 13 implies that $\ell-$ countL $^{\prime} \leq$ S-bins $^{\prime}+$ late-S-bins $^{\prime} \leq$ $3 s / 8-\ell / 4+1$ and

$$
\begin{aligned}
& \frac{2 \text {-Phase-Packer }(\sigma, s, \ell)-L}{\operatorname{OPT}(\sigma, s, \ell)} \leq 1+\frac{2\left(\ell-\operatorname{countL}^{\prime}-1\right)}{s+2 \ell} \leq 1+\frac{2(3 s / 8-\ell / 4)}{s+2 \ell} \\
& =1+\frac{s / 4+\ell / 2+s / 2-\ell}{s+2 \ell}<1+\frac{s / 4+\ell / 2}{s+2 \ell}=\frac{5}{4} .
\end{aligned}
$$

Similarly, if $s<6 \ell / 5$, then late-S-bins ${ }^{\prime}=0$, by Observation 1, so $\ell-$ countL $^{\prime} \leq$ S-bins $^{\prime} \leq s / 6+1$, by Lemma 13 and

$$
\begin{aligned}
& \frac{2 \text {-Phase-Packer }(\sigma, s, \ell)-L}{\operatorname{OPT}(\sigma, s, \ell)} \leq 1+\frac{2\left(\ell-\operatorname{count}^{\prime}-1\right)}{s+2 \ell} \\
& \leq 1+\frac{s / 3}{s+2 \ell}<1+\frac{2}{3+6 \ell / s} .
\end{aligned}
$$

Case 2: OPT has no items of size $S$ after $b^{\prime}$ In this case, Lemma 5 implies that, after bin $b^{\prime}$, OPT has $k=\left(s-\right.$ countS $\left.^{\prime}\right) / 2+\left(\ell-\right.$ countL $\left.^{\prime}\right)$ items of size $L$ remaining, so $O P T(\sigma, s, \ell)=X+O P T\left(\sigma^{\prime}, 0, k\right)$.

Since 2-Phase-Packer is reasonable, Lemma 10 implies that

$$
\begin{aligned}
& \text { 2-Phase-Packer }(\sigma, s, \ell)=X+2 \text {-Phase-Packer }\left(\sigma^{\prime}, s-\operatorname{count}^{\prime}, \ell-\operatorname{countL}^{\prime}\right) \\
& \leq X+O P T\left(\sigma^{\prime}, 0, k\right)+\left(\left(s-\operatorname{count} \mathrm{S}^{\prime}\right)+\left(\ell-\operatorname{count} \mathrm{L}^{\prime}\right)-k-1\right) L+M \\
& =O P T(\sigma, s, \ell)+\left(s-\operatorname{count} S^{\prime}\right) L / 2+M-L .
\end{aligned}
$$


By Proposition 6, $O P T(\sigma, s, \ell) \geq s S+\ell L \geq s L / 2+\ell L$.

When computing the competitive ratio, we will choose the additive constant to be at least $M$, so we subtract $M$ from 2-Phase-Packer's cost in the ratio. Thus,

$$
\begin{aligned}
\frac{2-\mathrm{Phase}-\mathrm{Packer}(\sigma, s, \ell)-M}{\operatorname{OPT}(\sigma, s, \ell)} & \leq \frac{\mathrm{OPT}(\sigma, s, \ell)+\left(s-\operatorname{count} \mathrm{S}^{\prime}\right) L / 2-L}{\operatorname{OPT}(\sigma, s, \ell)} \\
& \leq 1+\frac{\left(\left(s-\operatorname{count} \mathrm{S}^{\prime}\right) / 2-1\right) L}{s L / 2+\ell L} \\
& =1+\frac{s-\operatorname{countS}^{\prime}-2}{s+2 \ell} .
\end{aligned}
$$

It remains to bound $s-$ countS $^{\prime}-2$ in each of the three ranges for the ratio $s / \ell$.

First, suppose that $\ell \leq s / 2$. Immediately after bin $b^{\prime}$, OPT has packed at most 2L-bins' additional items of size $S$ than 2-Phase-Packer, so $s \leq$ count $^{\prime}+2 \mathrm{~L}$-bins' . When Phase 1 is finished, countL $=\lfloor\ell / 2\rfloor$, since countL either changes by 0 or 1 each iteration. After Phase 1, L-bins does not change. By Observation 1, L-bins $\leq$ countL. Hence L-bins' $\leq\lfloor\ell / 2\rfloor \leq \ell / 2$. Thus,

$$
\begin{aligned}
\frac{2 \text {-Phase-Packer }(\sigma, s, \ell)-M}{\operatorname{OPT}(\sigma, s, \ell)} & \leq 1+\frac{s-\text { countS }^{\prime}-2}{s+2 \ell}<1+\frac{2 \text { L-bins }^{\prime}}{s+2 \ell} \\
& \leq 1+\frac{\ell}{s+2 \ell}=1+\frac{1}{2+s / \ell} .
\end{aligned}
$$

Next, consider the case where $s / 2<\ell \leq 5 s / 6$. If $\operatorname{countS}^{\prime} \geq 3 s / 4-\ell / 2-2$, then $s-$ countS $^{\prime}-2 \leq s-3 s / 4+\ell / 2=(s+2 \ell) / 4$. Otherwise, count $S^{\prime} \leq 3 s / 4-\ell / 2-2<$ $\lfloor 3 s / 4-\ell / 2\rfloor$, so Phase 1 is not completed when bin $b^{\prime}$ is packed. Therefore, the number of bins in which 2-Phase-Packer packed one item of size $L$, but OPT packed

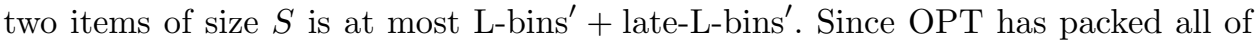
the items of size $S, s \leq$ countS $^{\prime}+2$ L-bins $^{\prime}+2$ late-L-bins' ${ }^{\prime}$. By Observation 1 and Lemma 14, 2S-bins' +2 late-S-bins ${ }^{\prime} \leq$ countS $^{\prime}$, L-bins $^{\prime} \leq\left\lfloor(10 \ell-3 s)\right.$ S-bins $\left.^{\prime} /(s+2 \ell)\right\rfloor+$ 1, and late-L-bins ${ }^{\prime} \leq$ late-S-bins $^{\prime}+1$. From the algorithm, S-bins ${ }^{\prime} \leq\lfloor(s+2 \ell) / 16\rfloor$, since S-bins increases by at most one each iteration until it reaches $\lfloor(s+2 \ell) / 16\rfloor$ and does not increase thereafter. Hence,

$$
\begin{aligned}
& s-\text { count }^{\prime}-2 \leq 2\left(\text { L-bins }^{\prime}+\right.\text { late-L-bins } \\
&
\end{aligned}
$$

Therefore,

$$
\frac{2 \text {-Phase-Packer }(\sigma, s, \ell)-M}{\operatorname{OPT}(\sigma, s, \ell)} \leq 1+\frac{s-\operatorname{countS}^{\prime}-2}{s+2 \ell} \leq 1+\frac{(s+2 \ell) / 4}{s+2 \ell}=\frac{5}{4} .
$$

When $5 s / 6<\ell$, we show that countS $\geq\lfloor s / 3\rfloor$. Suppose not. Then Phase 1 is not completed when bin $b^{\prime}$ is packed, so the number of bins in which 2-Phase-Packer 
packed one item of size $L$, but OPT packed two items of size $S$ is at most L-bins'. Since OPT has packed all of the items of size $S, s \leq$ countS $^{\prime}+2$ L-bins' ${ }^{\prime}$. By Lemma 14 and Observation 1, L-bins ${ }^{\prime} \leq 2$ S-bins $^{\prime}+1 \leq$ countS $^{\prime}+1$. Hence $s \leq 3$ countS $^{\prime}+2$ and count $S^{\prime} \geq(s-2) / 3$. Since count $S^{\prime}$ is an integer and $(s-2) / 3>(s-3) / 3 \geq\lfloor s / 3\rfloor-1$ it follows that count $S^{\prime} \geq\lfloor s / 3\rfloor$, which is a contradiction.

Thus, countS $\geq\lfloor s / 3\rfloor$, so $s-$ countS $^{\prime}-2 \leq\lceil 2 s / 3\rceil-2<2 s / 3$ and

$$
\begin{aligned}
\frac{2-\operatorname{Phase}-\operatorname{Packer}(\sigma, s, \ell)-M}{\operatorname{OPT}(\sigma, s, \ell)} & \leq 1+\frac{s-\operatorname{count}^{\prime}-2}{s+2 \ell} \\
& <1+\frac{2 s / 3}{s+2 \ell} \\
& =1+\frac{2}{3+6 \ell / s} .
\end{aligned}
$$

Case 3: 2-Phase-Packer runs out of items of size $L$ before OPT runs out of anything Suppose that, in this instance, 2-Phase-Packer packs its last item of size $L$ in bin $b^{\prime}$, but, after bin $b^{\prime}$, OPT has $i>0$ items of size $S$ and $j>0$ items of size $L$ that are unpacked. Then, by Lemma 5 , 2-Phase-Packer has $s-$ countS $^{\prime}=i+2 j$ unpacked items of size $S$.

Note that $j \leq$ L-bins' $^{\prime}+$ late-L-bins', since the only bins where 2-Phase-Packer had a choice and packed one item of size $L$ where OPT possibly did not are the bins contributing to the values of L-bins and late-L-bins. By Lemma 11, 2-Phase-Packer $(\sigma, s, \ell)=X+2$-Phase-Packer $\left(\sigma^{\prime}, i+2 j, 0\right) \leq X+\operatorname{OPT}\left(\sigma^{\prime}, i, j\right)+$ $(j-1) L+M=\operatorname{OPT}(\sigma, s, \ell)+(j-1) L+M$. By Proposition [6. OPT $(\sigma, s, \ell) \geq$ $s S+\ell L \geq s L / 2+\ell L$. In computing the competitive ratio, we will let the additive constant be $M$, so we will subtract this value from 2-Phase-Packer's cost in the ratio.

Thus,

$$
\begin{aligned}
\frac{2-\mathrm{Phase}-\operatorname{Packer}(\sigma, s, \ell)-M}{\operatorname{OPT}(\sigma, s, \ell)} & \leq \frac{\operatorname{OPT}(\sigma, s, \ell)+(j-1) L}{\operatorname{OPT}(\sigma, s, \ell)} \\
& =1+\frac{(j-1) L}{\operatorname{OPT}(\sigma, s, \ell)} \\
& \leq 1+\frac{(j-1) L}{s L / 2+\ell L} \\
& =1+\frac{2 j-2}{s+2 \ell}
\end{aligned}
$$

We now bound $2 j$ in each of the three cases of the algorithm.

Suppose $\ell \leq s / 2$. Phase 1 has completed, since all items of size $L$ have been packed by 2 -Phase-Packer. L-bins' is at most the value of countL when Phase 1 completed, 
which is $\lfloor\ell / 2\rfloor$. Thus, $2 j \leq 2 \mathrm{~L}$-bins ${ }^{\prime} \leq 2\lfloor\ell / 2\rfloor \leq \ell$. Hence,

$$
\begin{aligned}
\frac{2 \text {-Phase-Packer }(\sigma, s, \ell)-M}{\operatorname{OPT}(\sigma, s, \ell)} & \leq 1+\frac{2 j}{s+2 \ell} \\
& \leq 1+\frac{\ell}{s+2 \ell} \\
& \leq 1+\frac{1}{2+s / \ell} .
\end{aligned}
$$

Next, suppose that $6 \ell / 5 \leq s<2 \ell$. If countS $\geq 3 s / 4-\ell / 2-2$, then $2 j-2=$ $s-$ count $^{\prime}-i-2<s / 4+\ell / 2=(s+2 \ell) / 4$. Otherwise, count $S^{\prime}<3 s / 4-\ell / 2-2<$ $\lfloor 3 s / 4-\ell / 2\rfloor$, so Phase 1 is not done when bin $b^{\prime}$ is packed. Therefore, since $j$ is at most equal to the number of bins in which 2-Phase-Packer packed one item of size $L$, but OPT did not, $j \leq$ L-bins $^{\prime}+$ late-L-bins'. By Observation 11 and Lemma 14. 2 S-bins ${ }^{\prime}+2$ late-S-bins' $\leq$ countS $^{\prime}$, L-bins $^{\prime} \leq\left\lfloor(10 \ell-3 s)\right.$ S-bins $\left.^{\prime} /(s+2 \ell)\right\rfloor+1$, and late-L-bins ${ }^{\prime} \leq$ late-S-bins ${ }^{\prime}+1$. From the algorithm, S-bins ${ }^{\prime} \leq\lfloor(s+2 \ell) / 16\rfloor$, since S-bins' increases by at most one each iteration until it reaches $\lfloor(s+2 \ell) / 16\rfloor$ and does not increase thereafter. Hence $2 j-2 \leq 2$ L-bins $^{\prime}+$ late-L-bins $\left.^{\prime}-1\right) \leq 2(1+$ $(10 \ell-3 s)$ S-bins $^{\prime} /(s+2 \ell)+1+$ late-S-bins $\left.{ }^{\prime}-1\right)=2$ (S-bins $^{\prime}+$ late-S-bins ${ }^{\prime}+(8 \ell-$ $4 s)$ S-bins $\left.^{\prime} /(s+2 \ell)+1\right) \leq$ countS $^{\prime}+2(8 \ell-4 s) / 16+2<3 s / 4-\ell / 2-2+\ell-s / 2+2=$ $(s+2 \ell) / 4$. Hence,

$$
\begin{aligned}
\frac{2-\mathrm{Phase}-\operatorname{Packer}(\sigma, s, \ell)-M}{\operatorname{OPT}(\sigma, s, \ell)} & \leq 1+\frac{2 j-2}{s+2 \ell} \\
& \leq 1+\frac{(s+2 \ell) / 4}{s+2 \ell}=\frac{5}{4} .
\end{aligned}
$$

Now, suppose that $6 \ell / 5>s$. If Phase 1 is done, then countS $\geq\lfloor s / 3\rfloor>s / 3-1$, so $2 j=s-$ countS $^{\prime}-i \leq s-s / 3+1-i \leq 2 s / 3$. Otherwise, count $\mathrm{S}^{\prime}<\lfloor s / 3\rfloor$. Then, since count $^{\prime} \geq 2$ S-bins' ${ }^{\prime}$, it follows from Lemma 14 that $j \leq$ L-bins' $^{\prime} \leq 2$ S-bins $^{\prime}+1 \leq$ count $S^{\prime}+1 \leq\lfloor s / 3\rfloor \leq s / 3$. Hence,

$$
\begin{aligned}
\frac{2-\operatorname{Phase}-\operatorname{Packer}(\sigma, s, \ell)-M}{\operatorname{OPT}(\sigma, s, \ell)} & \leq 1+\frac{2 j}{s+2 \ell} \\
& \leq 1+\frac{2 s / 3}{s+2 \ell} \\
& <1+\frac{2}{3+6 \ell / s} .
\end{aligned}
$$

Case 4: 2-Phase-Packer runs out of items of size $S$ before OPT runs out of anything Suppose that, in this instance, 2-Phase-Packer packs its last item of size $S$ in bin $b^{\prime}$, but, after bin $b^{\prime}$, OPT has $i>0$ items of size $S$ and $j>0$ items of size $L$ that are unpacked. Then, 2-Phase-Packer has $\ell$ - countL' unpacked items of size $L$, and, by Lemma 5, $2\left(\ell-\right.$ count $\left.^{\prime}\right)=i+2 j$. Note that, in this case, $\ell \geq i / 2+j \geq \frac{1}{2}+1$, so $\ell \geq 2$. 
Since 2-Phase-Packer is reasonable, Lemma 12 implies that

2-Phase-Packer $(\sigma, s, \ell)=X+R\left(\sigma^{\prime}, 0, \ell-\operatorname{countL}^{\prime}\right)$

$$
\begin{aligned}
& \leq X+\operatorname{OPT}\left(\sigma^{\prime}, i, j\right)+\min \{0, j-i\} S+\left(\ell-\operatorname{countL}^{\prime}-j\right) M \\
& =\operatorname{OPT}(\sigma, s, \ell)+\min \{0, j-i\} S+(i / 2) M .
\end{aligned}
$$

By Proposition [6 $\operatorname{OPT}(\sigma, s, \ell) \geq s S+\ell L \geq s L / 2+\ell L$. Thus,

$$
\begin{aligned}
& \frac{2 \text {-Phase-Packer }(\sigma, s, \ell)}{\operatorname{OPT}(\sigma, s, \ell)} \leq 1+\frac{(i / 2) M+\min \{0, j-i\} S}{s L / 2+\ell L} \\
& =1+\frac{i(2 L-1)+\min \{0, j-i\}(L+1)}{s L+2 \ell L} \\
& =1+\frac{L(i+\min \{i, j\})-i+\min \{0, j-i\}}{L(s+2 \ell)} \\
& \leq 1+\frac{i+\min \{i, j\}}{s+2 \ell} \text {. }
\end{aligned}
$$

First suppose $\ell \leq s / 2$. If Phase 1 has not ended, then the only bins where 2-Phase-Packer had a choice and packed two more items of size $S$ than OPT did are the bins contributing to the value of S-bins, so $i \leq$ 2S-bins'. By Lemma 14, S-bins $^{\prime} \leq$ L-bins $^{\prime} \leq$ countL' $^{\prime}$.

If $j \geq i$, then $2\left(\ell-\right.$ countL $\left.^{\prime}\right)=i+2 j \geq 3 i$, so $i \leq 2\left(\ell-\right.$ countL $\left.^{\prime}\right) / 3$. In this case, if countL' $\geq \ell / 4$, then $i \leq 2\left(\ell-\right.$ countL $\left.^{\prime}\right) / 3 \leq \ell / 2$. If not, since $\ell \geq 2$, countL $^{\prime}<\ell / 4 \leq(\ell-1) / 2 \leq\lfloor\ell / 2\rfloor$, so Phase 1 has not ended and $i \leq 2 S$-bins ${ }^{\prime} \leq$ $2 \mathrm{~L}$-bins ${ }^{\prime} \leq 2$ count $^{\prime}<\ell / 2$. Therefore,

$$
\frac{2 \text {-Phase-Packer }(\sigma, s, \ell)}{\operatorname{OPT}(\sigma, s, \ell)} \leq 1+\frac{2 i}{s+2 \ell} \leq 1+\frac{\ell}{s+2 \ell}=1+\frac{1}{2+s / \ell} .
$$

Otherwise, $j<i$. If countL' $\geq\lfloor\ell / 2\rfloor$, then $i+j=2\left(\ell-\right.$ countL $\left.^{\prime}\right)-j \leq 2(\ell-$ countL $\mathrm{L}^{\prime}-1 \leq 2\lceil\ell / 2\rceil-1 \leq \ell$. If not, countL' $<\lfloor\ell / 2\rfloor$, so Phase 1 has not ended, $i \leq 2$ S-bins ${ }^{\prime} \leq 2 \mathrm{~L}^{- \text {bins }^{\prime}} \leq 2$ countL $^{\prime}$, and $i+j=\ell-$ countL $^{\prime}+i / 2 \leq \ell$. Hence

$$
\frac{2 \text {-Phase-Packer }(\sigma, s, \ell)}{\operatorname{OPT}(\sigma, s, \ell)} \leq 1+\frac{i+j}{s+2 \ell} \leq 1+\frac{\ell}{s+2 \ell} \leq 1+\frac{1}{2+s / \ell} \text {. }
$$

So, suppose that $\ell>s / 2$. Then $i \leq 2$ (S-bins' + late-S-bins'), because the only bins where 2-Phase-Packer had a choice and packed two more items of size $S$ than OPT did are the bins contributing to the value of S-bins or late-S-bins. Since 2-Phase-Packer has run out of items of size $S$, Phase 1 has ended.

Now suppose that $s / 2<\ell \leq 5 s / 6$. If S-bins' ${ }^{\prime}+$ late-S-bins $^{\prime} \leq(s+2 \ell) / 16$, then $i+\min \{i, j\} \leq 2 i \leq 4\left(\right.$ S-bins $^{\prime}+$ late-S-bins $\left.^{\prime}\right) \leq(s+2 \ell) / 4$, so

$$
\frac{2 \text {-Phase-Packer }(\sigma, s, \ell)}{\operatorname{OPT}(\sigma, s, \ell)} \leq 1+\frac{s+2 \ell}{4(s+2 \ell)}=\frac{5}{4} \text {. }
$$


Otherwise, S-bins ${ }^{\prime}=\lfloor(s+2 \ell) / 16\rfloor$. By Lemma 14, L-bins' $\geq\left\lfloor c\left(\right.\right.$ S-bins $\left.\left.^{\prime}-1\right)\right\rfloor+$ 1 , where $1<c=\frac{10 \ell-3 s}{s+2 \ell} \leq 2$ and late-L-bins ${ }^{\prime} \geq$ late-S-bins'. Since L-bins $^{\prime}+$ late-L-bins ${ }^{\prime} \leq$ countL $^{\prime}$, it follows that

$$
\begin{aligned}
& i+\min \{i, j\} \leq i+j=\frac{i}{2}+\ell-\text { countL' }^{\prime} \\
& \leq \text { S-bins }^{\prime}+\text { late-S-bins }^{\prime}+\ell-\text { L-bins }^{\prime}-\text { late-L-bins }{ }^{\prime} \\
& \leq \ell+\text { S-bins }^{\prime}-\left\lfloor c\left(\text { S-bins }^{\prime}-1\right)\right\rfloor-1 \\
& <\ell+\text { S-bins }^{\prime}-c\left(\text { S-bins }^{\prime}-1\right) \\
& =\ell+(1-c)\lfloor(s+2 \ell) / 16\rfloor+c \\
& \leq \ell+(1-c)((s+2 \ell) / 16-1)+c \\
& =\ell+\left(1-\frac{10 \ell-3 s}{s+2 \ell}\right) \frac{s+2 \ell}{16}+2 c-1 \\
& =(4 s+8 \ell) / 16+2 c-1 \text { and } \\
& \frac{2 \text {-Phase-Packer }(\sigma, s, \ell)-3}{\operatorname{OPT}(\sigma, s, \ell)} \leq 1+\frac{(4 s+8 \ell) / 16}{s+2 \ell}=\frac{5}{4} \text {. }
\end{aligned}
$$

Finally, suppose that $5 s / 6<\ell$. When Phase 1 finished, 2S-bins $\leq$ countS $\leq\lfloor s / 3\rfloor+$ 2. Thereafter, S-bins does not change. Hence, $i \leq 2$ S-bins' $\leq s / 3+2$ and

$$
\frac{2 \text {-Phase-Packer }(\sigma, s, \ell)-4}{\operatorname{OPT}(\sigma, s, \ell)} \leq 1+\frac{2 i-4}{s+2 \ell} \leq 1+\frac{2 s / 3}{s+2 \ell}=1+\frac{2}{3+6 \ell / s} .
$$

\section{A Relaxed Version of Grid Scheduling}

In the definition of the Grid Scheduling problem, we restricted the online algorithms so that after each bin is packed, no unpacked item fits into its remaining space. In this section, we consider a relaxed version of Grid Scheduling without this restriction. Specifically, in this version, a packing can be feasible even if there is a bin which has enough empty space to contain one of the items packed in a later bin. We show that the restriction does not make the problem easier. This may be useful for narrowing the gap between the upper and lower bounds for the Grid Scheduling problem. The results below imply that the lower bounds and the properties of optimal packings, which were proved in Sections 3 and 4, also hold for the relaxed version of Restricted Grid Scheduling.

We begin with a lemma that concerns packings of subsets of the items. If a bin, $b$, in a packing contains only one item, $i$, and there is at least one item in a later bin which is no larger than $b$, removing the item $i$ from the sequence might require substantial repacking to produce a feasible packing. However, this lemma shows that there is a feasible repacking that uses a (not necessarily proper) subset of the bins used in the original packing. 
Lemma 16. For any packing $p$ of a finite set of items $I$ into a sequence of bins $\sigma$ and any item $i \in I$, there is a packing $p^{\prime}$ of $I-\{i\}$ into $\sigma$ that uses a subset of the bins used by $p$. If $p$ was produced by an online algorithm, then there exists an online algorithm that produces $p^{\prime}$.

Proof. If $|I|=1$, the claim holds since, after removing one item, no bins are used. Let $p$ be any packing of a set $I$, with at least two items, into a sequence of bins $\sigma=\left\langle b_{1}, b_{2}, \ldots, b_{m}\right\rangle$. Assume the claim is true for any smaller set of items.

Let $b_{k}$ be the bin in which $p$ packs item $i$ and let $J \subseteq I-\{i\}$ be the set of items $p$ packs into later bins. If $p$ packs more than one item in bin $b_{k}$ or all items in $J$ are larger than size $\left(b_{k}\right)$, then the packing $p^{\prime}$ of $I-\{i\}$ that packs every item into the same bins as $p$ does is feasible.

Otherwise, there is an item $j \in J$ that is no larger than $b_{k}$. Let $q$ be the packing of $J$ into $\sigma^{\prime}=\left\langle b_{k+1}, \ldots, b_{m}\right\rangle$ that packs each item of $J$ into the same bin as $p$ does. Since the claim holds for any smaller set of items, there is a packing $q^{\prime}$ of $J-\{j\}$ that uses a subset of the bins used by $q$. Let $p^{\prime}$ be the packing of $I-\{i\}$ that packs the items of $I-(J \cup\{i\})$ into bins $b_{1}, \ldots, b_{k-1}$ the same as $p$ does, packs item $j$ into bin $b_{k}$, and packs the items of $J-\{j\}$ into bins $b_{k+1}, \ldots, b_{m}$ the same as $q^{\prime}$ does.

Now suppose that the packing $p$ was produced online by an algorithm $\mathbb{A}$. To produce $p^{\prime}$, an online algorithm $\mathbb{A}^{\prime}$, given the set of items $I-\{i\}$, could simulate $\mathbb{A}$ on the set of items $I-\{i\}$ together with an imaginary copy of item $i$, until it is about to pack $i$ into bin $b_{k}$. If $\mathbb{A}$ will pack more than one item into $b_{k}$ or the items that $\mathbb{A}$ will pack into later bins are all larger than $\operatorname{size}\left(b_{k}\right)$, then $\mathbb{A}^{\prime}$ continues to simulate $\mathbb{A}$ as if it had packed item $i$ into bin $b_{k}$. Otherwise, let $j$ be an unpacked item that is no larger than $b_{k}$ and let $J^{\prime}$ be the set of the other unpacked items. Since $\mathbb{A}$ produces a feasible packing $q$ of $J^{\prime} \cup\{j\}$ into $\sigma^{\prime}$, the induction hypothesis implies that there is an online algorithm $\mathbb{A}^{\prime \prime}$ that produces the feasible packing $q^{\prime}$. Then $\mathbb{A}^{\prime}$ packs item $j$ into bin $b_{k}$ and simulates $\mathbb{A}^{\prime \prime}$ on the set of remaining items $J^{\prime}$.

Hence, the claim is true for $I$. By induction, the claim is true for all finite sets of items $I$.

A bin in a packing is wasteful if its empty space is at least as large as some unpacked item or some item packed in a bin that occurs later in the sequence. In other words, when this bin was packed, there was an extra item that could have been added to it, but was not. A packing is thrifty if it contains no wasteful bins. The original version of the problem has the restriction that all feasible packings must be thrifty.

The following result shows that we may ignore algorithms which do not produce thrifty packings. 
Lemma 17. For any packing $p$ of a finite set of items $I$ into a sequence of bins $\sigma$, there is a thrifty packing $p^{\prime}$ of I into $\sigma$ using a subset of the bins used by $p$. If $p$ was produced by an online algorithm, then there exists an online algorithm that produces $p^{\prime}$.

Proof. Any packing of a set with at most one item is also thrifty. Let $I$ be a finite set with at least two items and assume the claim is true for any smaller set of items.

Let $p$ be any packing of $I$ into a sequence of bins $\sigma=\left\langle b_{1}, b_{2}, \ldots, b_{m}\right\rangle$. Suppose $p$ is not thrifty. Then it has at least one wasteful bin. Let $b_{k}$ be the first wasteful bin in $p$, let $e$ be the amount of empty space in $b_{k}$, and let $J \subseteq I$ be the set of items $p$ packs into later bins. Since $p$ is feasible, $b_{k}$ is nonempty, so $J \subsetneq I$. Since $b_{k}$ is wasteful, there is some item $i \in J$ of size at most $e$.

Let $q$ be the packing of $J$ into $\sigma^{\prime}=\left\langle b_{k+1}, \ldots, b_{m}\right\rangle$ that packs each item of $J$ into the same bin as $p$ does. By Lemma 16, there is a packing $q^{\prime}$ of $J-\{i\}$ into $\sigma^{\prime}$ that uses a subset of the bins used by $q$. Let $b$ be a bin of size $e$, let $\sigma^{\prime \prime}=\left\langle b, b_{k+1}, \ldots, b_{m}\right\rangle$, and let $q^{\prime \prime}$ be the packing of $J$ into $\sigma^{\prime \prime}$ that packs item $i$ into bin $b$ and packs each item of $J-\{i\}$ into the same bin that $q^{\prime}$ does.

Since $J \subsetneq I$, it follows by the induction hypothesis that there is a thrifty packing $p^{\prime \prime}$ of $J$ into $\sigma^{\prime \prime}$ using a subset of the bins used by $q^{\prime \prime}$. Note that $p^{\prime \prime}$ is feasible and $i \in J$, so $p^{\prime \prime}$ uses bin $b$. Moreover, every item $p^{\prime \prime}$ packs in later bins is smaller than the empty space in $b$.

Let $p^{\prime}$ be the packing of $I$ into $\sigma$ that packs each item in $I-J$ into the same bin as $p$, adds to bin $b_{k}$ the items packed by $p^{\prime \prime}$ into bin $b$, and packs bins $b_{k+1}, \ldots, b_{m}$ as $p^{\prime \prime}$ packed them. Since the empty space $p^{\prime}$ leaves in bin $b_{k}$ is equal to the empty space $p^{\prime \prime}$ leaves in bin $b$, bin $b_{k}$ is not wasteful in $p^{\prime}$. Bins $b_{1}, \ldots, b_{k-1}$ are not wasteful in $p^{\prime}$, since they are not wasteful in $p$. Bins $b_{k+1}, \ldots, b_{m}$ are not wasteful in $p^{\prime}$, since they are not wasteful in $p^{\prime \prime}$. Therefore $p^{\prime}$ is a thrifty packing that uses a subset of the bins used by $p$. By induction, the claim is true for all finite sets of items.

Suppose $p$ was produced by an online algorithm $\mathbb{A}$. Since $q$ is also produced by $\mathbb{A}$, Lemma 16 implies that $q^{\prime}$ is produced by an online algorithm $\mathbb{A}^{\prime}$. Then $q^{\prime \prime}$ can be produced by an online algorithm that packs item $i$ in the first bin that arrives and thereafter simulates algorithm $\mathbb{A}^{\prime}$. By the induction hypothesis, it follows that $p^{\prime \prime}$ can be produced by an online algorithm $\mathbb{A}^{\prime \prime}$. Finally, an online algorithm that produces $p^{\prime}$ proceeds by packing each item using $\mathbb{A}$ until it creates a wasteful bin, then simulates $p^{\prime \prime}$ with the remaining items, treating the empty space in the wasteful bin as if it were the first bin to arrive, followed by the rest of the sequence.

The previous lemma shows that restricting attention to thrifty packings is also sufficient for the lower bound. In other words, proving a lower bound on the competitive ratio for the class of thrifty packings then immediately implies the same 
lower bound for all packings. It eliminates the possibility that there is an online packing that is not thrifty and more efficient than any online thrifty packing. The following corollary shows that there is also an optimal (offline) packing which is thrifty.

Corollary 18. For any optimal packing of a set of items into a sequence of bins, there is an optimal thrifty packing of those items into that sequence using the same set of bins.

Proof. Let $p$ be an optimal packing of a set of items $I$ into a sequence of bins $\sigma$. Since $p$ is feasible, Lemma 17 implies that there is a thrifty packing packing $q$ of $I$ into $\sigma$ using a subset of the bins used by $p$. Thus $\operatorname{cost}(q) \leq \operatorname{cost}(p)$. But $p$ is optimal, so $\operatorname{cost}(q)=\operatorname{cost}(p)$. Hence $q$ is optimal and uses the same set of bins that $p$ uses $\square$

\section{Conclusions and Open Problems}

Note that the upper bound uses the additive constant in the definition of the competitive ratio, assuming that $M$ is a constant. As mentioned earlier, this bound on $M$ is necessary for the general Grid Scheduling problem or no algorithm is competitive. One would like to normalize and assume that $M=1$, since the lower bound holds with this assumption. The upper bound proof fails in Case 3 with this assumption because Lemma 11 fails. The proof uses the fact that the empty space in a bin containing exactly one item of size $S$ is at most $S-1=L-S$ (if that item is not the last). Thus, it implicitly assumes that there is no bin size between $L$ and $2 S$. Without this assumption, the term $\left(k-s^{\prime}-\ell^{\prime}-1\right) L$ becomes arbitrarily close to $\left(k-s^{\prime}-\ell^{\prime}-1\right) 2 S$, increasing the bounds on the competitive ratio by a factor of $2 S / L$. To obtain a tight upper bound without this assumption, one would need to change the algorithm, choosing other values for the ratios defined by the macros in 2-Phase-Packer. These values are defined using the values of $k$ computed in the lower bound proof in Theorem [1. We expect that simply modifying the constants in the macros would give an algorithm with the same upper bound, but an even more complicated analysis.

We have shown that varying the proportion of items of size $S$ to items of size $L$ does not lead to a larger competitive ratio if the maximum bin size is at most $4 S-3$. This may also be the case for an arbitrary maximum bin size, but there are complications. First, the invariant $s+2 \ell=s^{\prime}+2 \ell^{\prime}$, which holds until either OPT or the reasonable packing runs out of one item type, is very heavily used. For example, in Lemma 10 it is used to show that the reasonable packing runs out of large items before OPT does. With larger bins, the invariant no longer holds in some cases. For example, if some bin has size $S \cdot L$, one packing could contain $L$ items of size $S$, while the other packing contains $S$ items of size $L$. In addition, it may be an advantage to have mixed bins which contain more than one item of size $S$ and more 
than one item of size $L$. So, when bins can be large, one needs to consider how to maintain a good ratio of items of size $L$ to items of size $S$, as they are packed. We conjecture that maintaining the ratios specified in 2-Phase-Packer is sufficient.

One could also consider the Grid Scheduling problem for two bin sizes, $S$ and $L \neq 2 S-1$. For example, when $L$ is a multiple of $S$, then the algorithm of Example 2 , which always uses as many items of size $L$ as possible, is optimal and, hence, has competitive ratio 1 . It would be interesting to see if changing the ratio of $S$ to $L$ could improve the lower bound. We conjecture that it does not.

For the general problem, where there could be more than two sizes of items, we would like to close the gap between our lower bound and the upper bound of $\frac{13}{7}$ in [2].

Finally, it would be interesting to consider the competitive ratio of randomized algorithms for the Grid Scheduling problem or Restricted Grid Scheduling problem against an oblivious adversary.

\section{Acknowledgements}

Joan Boyar was supported in part by the Villum Foundation and the Danish Council for Independent Research, Natural Sciences (FNU). Part of this work was carried out while she was visiting the University of Waterloo and the University of Toronto.

Faith Ellen was supported in part by the VELUX Foundation and the Natural Science and Engineering Research Council of Canada (NSERC). Part of this work was carried out while she was visiting the University of Southern Denmark.

\section{References}

[1] A. Borodin and R. El-Yaniv, Online Computation and Competitive Analysis (Cambridge University Press, 1998).

[2] J. Boyar and L. M. Favrholdt, Scheduling jobs on Grid processors, Algorithmica 57(4) (2010) 819-847.

[3] J. Boyar and L. M. Favrholdt, A new variable-sized bin packing problem, Journal of Scheduling 15 (2012) 273-287.

[4] A. Darling, L. Carey and W. Feng, The design, implementation, and evaluation of mpiBLAST, ClusterWorld Conference $\mathcal{E}$ Expo and the 4 th International Conference on Linux Cluster: The HPC Revolution 2003, (2003).

[5] L. Epstein, L. M. Favrholdt and A. Levin, Online variable-sized bin packing with conflicts, Discrete Optimization 8(2) (2011) 333-343.

[6] L. Epstein and A. Levin, More online bin packing with two item sizes, Discrete Optimization 5(4) (2008) 705-713.

[7] G. Gutin, T. R. Jensen and A. Yeo, On-line bin packing with two item sizes, Algorithmic Operations Research 1(2) (2006).

[8] A. R. Karlin, M. S. Manasse, L. Rudolph and D. D. Sleator, Competitive snoopy caching, Algorithmica 3(1) (1988) 79-119. 
[9] F. M. Liang, A lower bound for on-line bin packing, Inform. Process. Lett. 10 (1980) $76-79$.

[10] D. D. Sleator and R. E. Tarjan, Amortized efficiency of list update and paging rules, Comm. of the ACM 28(2) (1985) 202-208.

[11] B. Vinter, The architecture of the minimum intrusion grid (mig), The 28th Communicating Process Architectures Conference, CPA 2005, eds. J. Broenink, H. Roebbers, J. Sunter, P. Welch and D. Wood Concurrent Systems Engineering Series 63, (IOS Press, 2005), pp. 189-201.

[12] G. Zhang, A new version of on-line variable-sized bin packing, Discrete Applied Mathematics 72 (1997) 193-197. 